\title{
The cognition/metacognition tradeoff
}

\author{
David Rosenbaum, Moshe Glickman, Stephen M. Fleming \& Marius Usher
}

\begin{abstract}
Integration-to-boundary is an optimal decision algorithm that takes samples of evidence until the posterior reaches a decision boundary, resulting in the fastest decisions for a target accuracy. For example, integration-to-boundary achieves faster mean RT compared with taking a fixed number of samples that result in the same choice accuracy. Here we demonstrate that this advantage comes at a cost in metacognitive accuracy, generating a cognition-metacognition tradeoff. Using computational modeling, we show that integration to a fixed boundary results in less variability in evidence integration, and is less predictive of choice accuracy (i.e., it has lower metacognitive accuracy), compared with a collapsing boundary or a random timer strategy. We examine how the decision strategy affects metacognitive accuracy in three cross-domain experiments, in which all participants $(n=102$ students $)$ carried out two sessions that manipulated the response-mode protocol: free-response (evidence terminated by the subject's response) vs. interrogation (fixed number of evidence samples controlled by the experimenter, which is the same as in the free-response session). In both sessions the participants observe a sequence of evidence frames (presented at $2 \mathrm{~Hz}$ ) and report their choice and confidence. As predicted, the interrogation protocol (which prevents integration to boundary) enhances metacognitive accuracy. We also find that in the free-response sessions the participants integrated to a collapsing boundary - a strategy that achieves an efficient compromise between optimizing both choice and metacognitive accuracy.
\end{abstract}

\section{Corresponding Author:}

David Rosenbaum

Email: davidros28@gmail.com

School of Psychological Science Tel-Aviv University, Tel-Aviv, Israel 
While cognition refers to the set of processes via which we make judgments about the world (e.g., deciding if a stock's value is likely to raise based on a sequence of returns ), metacognition refers to judgments we make about our own cognition (e.g., how confident we are in our decision to purchase this stock). Confidence is a canonical example of such a metacognitive judgment, with previous research demonstrating that humans can both estimate their decision confidence in a way that is reliably related to cognitive performance, and rely on confidence to modulate further cognition (Bahrami et al., 2010; Balsdon, Wyart \& Mamassian, 2020; Carlebach \& Yeung, 2020; Desender, Boldt, Verguts \& Donner, 2019; Hainguerlot, Vergnaud \& de Gardelle, 2018; Rollwage et al., 2020; Sanders, Hangya \& Kepecs, 2016; van den Berg, Zylberberg, Kiani, Shadlen \& Wolpert, 2016). The extent to which confidence tracks variation in objective performance is known as "confidence resolution" or "metacognitive sensitivity" (Fleming \& Lau, 2014).

In turn, research in the last few decades has converged on integration-to-boundary as an efficient, Bayesian mechanism for evidence-based decision making (Bogacz et al., 2006; Ratcliff, Smith, Brown, \& McKoon, 2016; Teodorescu \& Usher, 2013), which allows participants to decide not only what decision to make, but also how long to keep gathering evidence before committing to a particular choice (Figure 1B). By integrating to a boundary, one improves the signal to noise ratio, resulting in decisions with the fastest RT for a given accuracy level (Wald \& Wolfowitz, 1948; Bogacz et al., 2006). Moreover, integration-to-boundary is a key mechanism in most choice-RT models which account for key experimental data patterns, such as the shape of the RT distribution and its dependency on the strength of evidence, as well as the speed-accuracy tradeoff (Ratcliff et. al, 2016; Teodorescu \& Usher, 2013).

There is less agreement on the mechanisms supporting metacognitive judgments of confidence about such decisions (Yeung \& Summerfield, 2014). While various confidence mechanisms have been proposed, they differ drastically in their nature, from Vickers' balance of evidence (BOE), which is based on a non-normative race model of choice (Vickers, 1979; see also, De Martino et al., 2013; Merkle \& Van Zandt, 2006; Reynolds, Kvam, Osth \& Heathcote, 2020) to the more recent two-stage dynamic signal detection theory (2DSD) model (Pleskac \& Busemeyer, 2010), which is based on a normative, integration-to-boundary choice model. One problem facing the latter type of models is the 
following: If evidence keeps being collected until the integrated evidence reaches a constant bound (corresponding to the expected posterior probability; Fig. 1B) there is little variability left (between trials) that can signal variations in objective accuracy. In other words, these models suggest that confidence should be similar for decisions that terminate at the same bound ${ }^{1}$. To deal with this problem, normative confidence models such as 2DSD introduced the idea of post-decision integration (Pleskac \& Busemeyer, 2010; Moran, Teodorescu \& Usher, 2015), according to which confidence is based on the consistency with which some extra evidence (collected after making the decision but before the confidence is signaled) supports the decision. Other confidence models account for variations in confidence in additional ways, for example, by relying on decision time as a proxy for confidence (Zakay \& Tuvia, 1988), in addition to a BOE mechanism (Kiani et al., 2014), or by assuming that the decision itself is determined by a race between multiple confidence accumulators (Ratcliff \& Starns, 2009; for a recent review and discussion of normative models see Calder-Travis, Bogacz \& Yeung, 2020).

A broader consideration of such frameworks suggests that humans face a cognitive/metacognitive tradeoff with regards to the normative aspects of their decisions and confidence judgments. When faced with a stream of stochastically fluctuating evidence, an observer can integrate the evidence to a decision boundary - a fixed boundary is optimal for choices with a fixed difficulty level, corresponding to the sequential probability ratio test (SPRT; Wald, 1948) - optimizing the decisions at the expense of metacognitive accuracy or confidence resolution (defined here as the difference between the confidence of correct and incorrect responses). Alternatively, an observer can adopt a less normative decision strategy, which allows her enough information to achieve an improved confidence resolution. The simplest such strategy is to dispose of integration to boundary altogether, in favor of taking a number of samples (either fixed or determined by a random timer that is independent of the decision) before committing to the decision. While this results in less optimal decisions, it allows the observer to rely on the Signal Detection Theory (SDT) framework, in which confidence corresponds to the distance from a criterion (Kepecs \& Mainen, 2012), thus providing a robust degree of confidence resolution (see Fig. 1).

\footnotetext{
${ }^{1}$ If evidence comes in discrete samples (as in our experiments) the evidence will not precisely end at the boundary, but may overshoot it, with a degree that could depend on choice accuracy. Thus a small amount of variability may remain to support above-chance confidence resolution. We show in Fig 3A that this resolution is much lower than that obtained with other methods.
} 
(A)

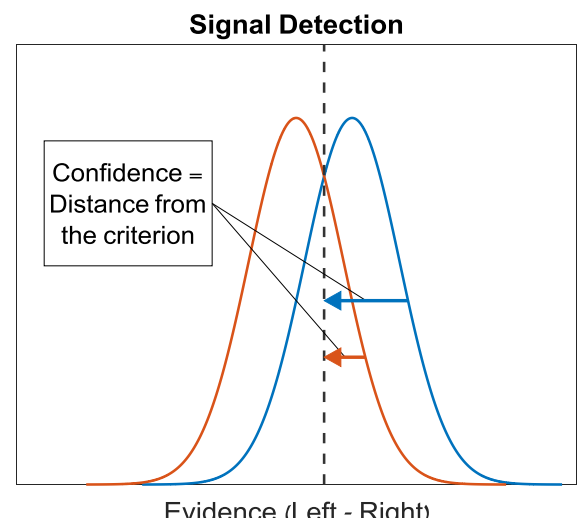

(B)

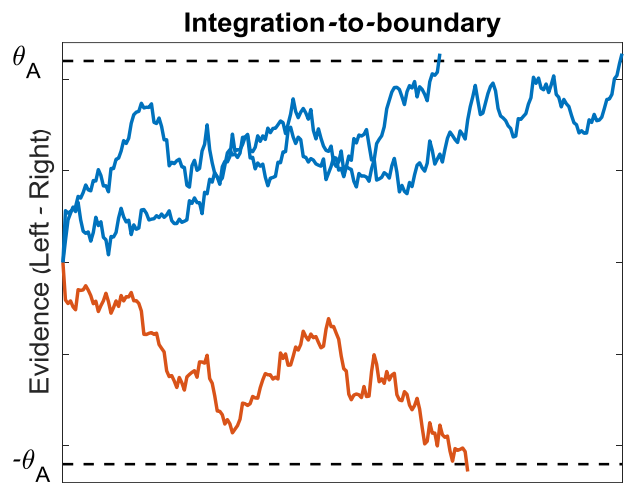

Fig. 1. (A) With a fixed number of samples, decisions and confidence can be generated using Signal Detection Theory, with the decision as a binary comparison of evidence to a criterion and confidence as the distance from criterion. Red/blue vertical arrows correspond to samples of correct/incorrect responses for which confidence is determined as the distance from criterion, indicated by the horizontal arrows. Resolution is positive as the further one is from criterion, both confidence and expected accuracy increase in tandem. (B) Integration to boundary model where the upper " $A$ " boundary is the correct response; three example trials are shown (2 correct and one error). Integration is terminated when the posterior probability reaches a constant threshold; this minimizes differences between trials in confidence, and therefore reduces confidence resolution. Dotted lines correspond to the response boundaries.

We report three experiments testing this idea. The first is a pilot $(N=17)$, the second is a preregistered experiment $(N=35)$, and the third is an experiment $(N=50)$ carried out during the review of this paper to rule out potential confounds. In all experiments, participants were asked to make decisions about fluctuating evidence, followed by confidence ratings. Each subject participated in two sessions. In the first session we used a free response protocol, in which the evidence is presented until the subject responds (this is thought to engage, in most subjects, an integration-to-boundary strategy). In the second session, we used an interrogation protocol, in which the evidence is presented for a predetermined number of samples and the response is requested after the evidence stream ends (Bogacz et al., 2006). Critically, each trial in the interrogation session has an equal number of frames (and the same average signal and noise) as in the corresponding trial from the free response session. To anticipate our results, we confirm the predicted tradeoff - metacognitive accuracy is higher (and choice accuracy is lower) - in the interrogation compared with the free response session. We start with a brief 
computational section that highlights the basis for our predictions, followed by the results of the experiments that examine how confidence is formed in the two paradigms.

\section{Computational methods: predictions on confidence-resolution}

Consider a task which presents sequences of pairs of 2-digits numbers, randomly sampled from two overlapping normal distributions that are unknown to the subjects (Fig. 2), who are required to choose which sequence - Left (L) or Right $(\mathrm{R})$ - is generated from the higher mean distribution.

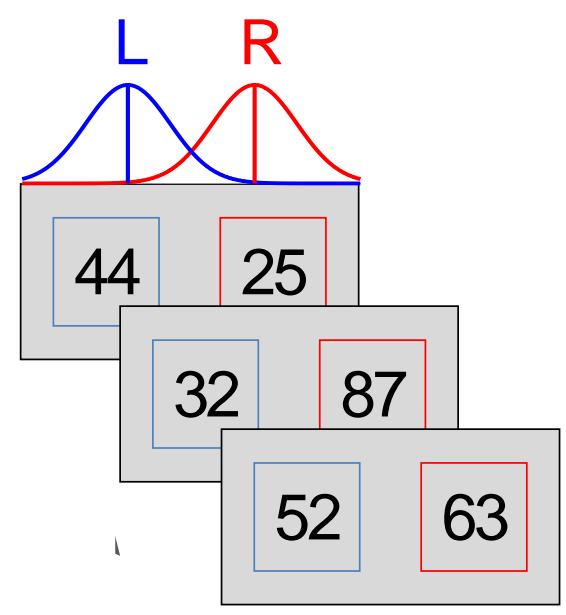

Fig. 2. Experimental paradigm in the free-response and interrogation sessions. In the free-response session sequences of two-digit numbers, selected from Gaussian distributions are presented at a rate of 2 pairs/sec, until the subject responds (left/right) to indicate the evidence stream that is larger on average. This trial is an example of a trial where the correct answer was " $R$ " (as the right-hand sequence is generated from a distribution with a higher mean). In the interrogation session the experimental paradigm was the same, except for the trial was terminated after a fixed number of samples.

We simulated two types of decision strategy: i) integration to a fixed boundary (SPRT) and ii) integration of a fixed number of samples (chosen so as to produce the same accuracy as in (i)). In (i) the amount of integrated evidence is by definition fixed by the constant boundary and exhibits little variability between trials (however, see footnote-1, and Fig 3A for simulation results showing that this produces very low confidence-accuracy correlations). We also considered the prominent 2DSD mechanism (Pleskac \& Busemeyer, 2010), which suggests that in such situations, confidence resolution can be improved by sampling post-decision evidence and examining the strength of its consistency with the decision. We implemented this in the simplest way, by taking one extra sample (following the integration to boundary decision). This extra sample can carry information that is consistent with the decision (leading to higher confidence) or inconsistent with it (leading to lower confidence; see Fig. 1 
in Pleskac \& Busemeyer, 2010, reproduced here as Fig.S4 in Suppl.). ${ }^{2}$ As shown in Fig. 3B, this results in an increase of the correlation between confidence and accuracy.

For the 'integration over a fixed number of samples' simulation (which can be naturally applied either in an interrogation task on in a free-response task, if evidence termination is based on a random timer) we determined confidence from all the evidence available in the trial and not from the last frame only. Here there are two distributions for the total evidence, one for trials where the rightward evidence stream has a greater mean, and one for trials where the leftward evidence stream has a greater mean (where the $\mathrm{x}$-axis corresponds to L-R evidence). We therefore relied on standard SDT approaches (e.g., Kepecs \& Mainen, 2012) to compute choice (as the sign of the sample relative to the decision criterion) and confidence as the normalized distance (min-max normalization) from the decision criterion (i.e., the y-axis that corresponds to 0-confidence; see Suppl. for details and Fig. 1A). In Fig 3, we plot example distributions of the normalized confidence obtained from simulations of the three decision rules: i) integration to boundary only (top panel), ii) integration to boundary, followed by one postdecision sample for confidence (middle panel), and a decision based on fixed number of samples (using SDT, lower panel). In all cases, we show this separately for correct (blue) and for incorrect responses (orange), and report the correlation between confidence and choice accuracy. Note that we present the results only for the case in which the left distribution has a higher mean than the right one (L-trials), as the other case $(\mathrm{R}>\mathrm{L}$ ) is symmetrical (see Suppl. for further details). As shown in Fig. 3, the interrogation protocol results in enhanced confidence resolution, as indicated by a higher correlation between confidence and accuracy ( $r=.30$ compared to $r=.17)$ and by a larger difference in (normalized) confidence of correct and incorrect responses. This result is an expected outcome of the fact that the total evidence has more relevant information about the likelihood of a decision being correct, than a single (post-decision) evidence frame. Note that in the absence of any post-decision evidence, the integrated evidence becomes similar in all trials (but see footnote-1), and the confidenceaccuracy correlation is even lower ( $\mathrm{r}=.07$, in Fig 3 top panel).

\footnotetext{
${ }^{2}$ The confidence was determined by normalizing (min-max normalization across all trials) the match of the extra-sample with the decision (see Supplement for further details).
} 

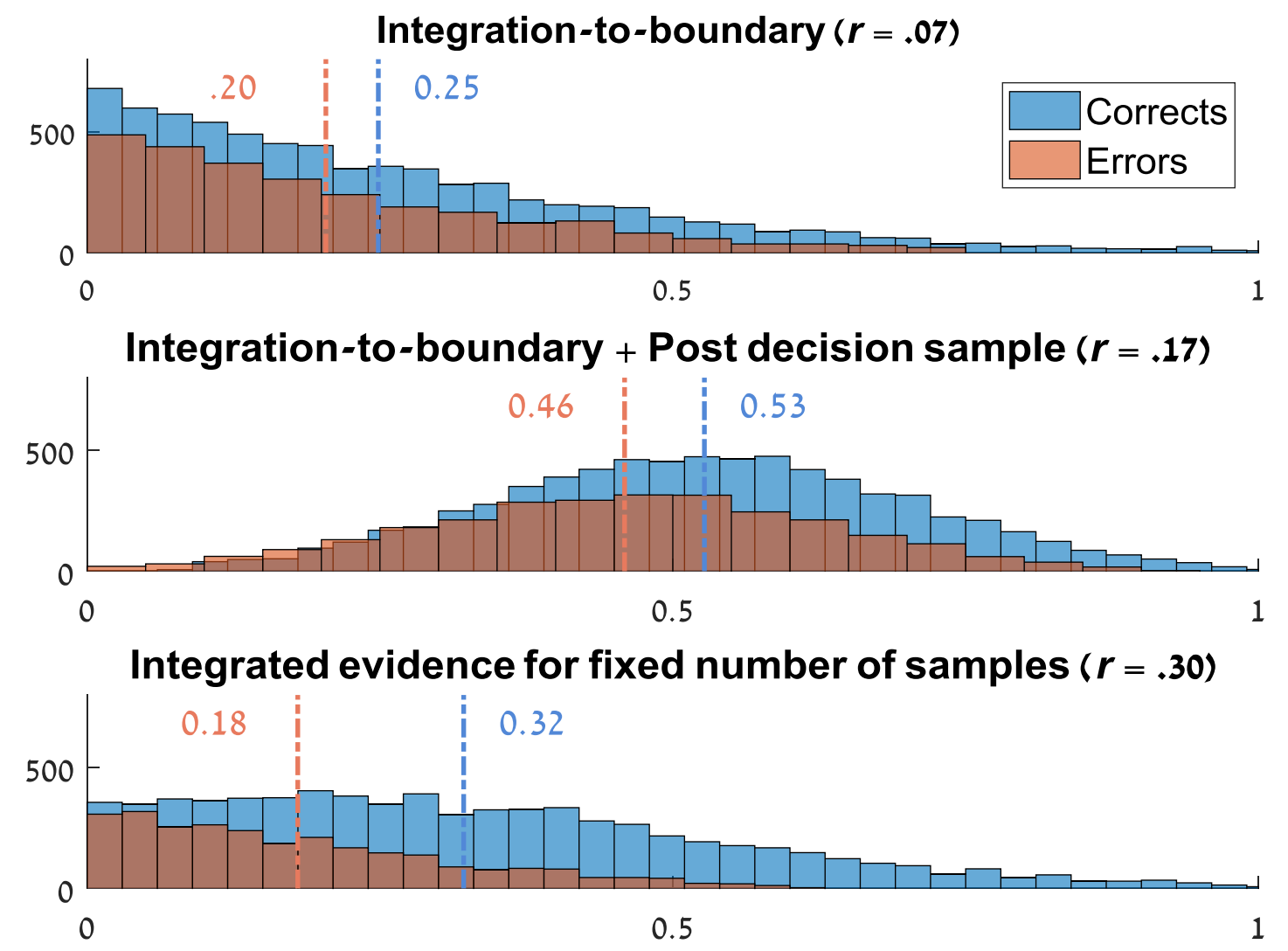

Normalized Confidence

Fig 3. Confidence distributions for three models of choice \& confidence. Each panel shows the distribution of normalized confidence levels for correct (blue) and incorrect (red) responses, and the correlation between confidence and choice accuracy. The upper and middle panels rely on integration to boundary to determine choice (in Free-response protocols). The two panels show the effects of two different rules for how confidence is computed: i) integrated evidence to boundary only (upper panel) or (integrated evidence + post-decision evidence sample; middle panel) to determine confidence. The lower panel computes both choice and confidence by applying SDT to the total evidence over a fixed number of samples that is selected to equalize accuracy levels of the free response and interrogation protocols. Here, confidence is determined using the SDT framework by calculating the absolute distance from the criterion (see Suppl. for details). Note the greater difference in mean confidence between correct and incorrect responses in the lower panel.

As we show in the Supplement, adopting a collapsing (rather than a fixed) boundary (Evans, Hawkins \& Brown, 2020; Glickman \& Usher, 2019; Palestro, Weichart, Sederberg \& Turner, 2018; Malhotra, Leslie, Ludwig \& Bogacz, 2017) in the free-response protocol allows agents to obtain a confidence resolution that is higher than that based on the last sample, but still lower that what could be obtained based on the total evidence (assuming the same number of evidence samples from the same distributions were used in the two cases). 


\section{Experimental Methods}

Each experiment presented participants with sequences of fluctuating evidence (pairs of numbers or lines sampled from overlapping Gaussians) at a rate of about 2 pairs/sec (see Fig. 2), and required them to decide which sequence originates from the higher of the two Gaussians. All participants carried out two sessions: a free response and an interrogation session. In the free response session, the evidence continued until response (or a deadline of 15 frames). In the interrogation session the evidence was presented for a fixed number of frames, which was equated (for each subject) to that of the free response session. This was done either on a trial by trial basis (Exp. 1-2) or on average (Exp. 3). For example, (in Exp. 1-2) if a subject responded in session 1 after 5 frames in trial '1' and after 9 frames in trial ' 2 ', in the second session that subject was presented with 5 frames in trial ' 1 ' and 9 frames in trial '2' (see Supplement for additional Methods).

Participants. A total of 102 undergraduates from Tel-Aviv University (68 females; age: M=25, range 21-35 years) participated in three experiments. The participants received course credit in exchange for taking part in the experiment. The experiments were approved by the ethics committee at TAU. The number of subjects in the first experiment (the pilot) was determined based on previous similar confidence studies (e.g., Pleskac \& Busemeyer, 2010). In Exp. $2(N=35)$ and Exp. $3(N=50)$, the number of subjects was determined through power analysis based on our effect sizes in Exp. 1 \& 2 respectively (we used higher power in Exp. 3, as this included few changes to the design; see Exp. 3 Methods).

\section{Experiments 1 \& 2}

Procedure and design. All experiments used the same experimental paradigms (see Fig. 2). Each trial began with a fixation point followed by two rapidly changing $(2 \mathrm{~Hz})$ sequences of numerical values. Participants were instructed to indicate which sequence was drawn from a distribution with a higher mean. Critically, we used a free response paradigm in the first session in which the participant's decision terminates the trial, and an interrogation paradigm in the second session in which participants had to wait till the sequences end and only then take a decision. The two sessions types were manipulated within participants and the free response session was always presented first. The number of samples in each trial in the interrogation session was determined according to the number of samples

the participant used in the free response session at the corresponding trial. A small difference between experiment 1 and experiment 2 was that in experiment 1 , the number of samples in each trial for the 
interrogation session was always equal to the corresponding trial from the free response session. In experiment 2, the number of samples in the interrogation session was either equal or equal minus 1 (n or $n-1)$ depending on the reaction time within the last sample ( $R T \geq 250 m s=n, R T>250=n-1$ ). This change was made due to our assumption that trials in which participants responded faster than $250 \mathrm{~ms}$ were trials in which the last sample was not used to guide the decision, but could be used to inform confidence. The stimuli were sampled from overlapping Gaussian distributions with $\sigma$ of 10. In the free response sessions, all participants started with the same means of the two Gaussians $\left(\mu_{1}=52\right.$ vs. $\mu_{2}=$ 46). In order to keep general performance between $70 \%-90 \%$ correct for all participants, $\mu_{2}$ increased in steps of 1 following blocks of 30 trials with performance higher than 90\% accuracy, and decreased in steps of 1 following blocks with performance lower than 70\% accuracy. Each session included 5 blocks each of 30 trials and the stimulus difficulty for each block of the interrogation session was equated to the corresponding block of the free response session.

\section{Results}

\section{Experiment 1}

Choice accuracy in each session and three measures of confidence resolution are reported in Table 1 as averages over subjects. For the free-response session, accuracy corresponds to the fraction of trials in which subjects selected the stream generated from the higher Gaussian. Thus, if a subject responds after a single frame selecting the larger of the two numbers presented, this counts as an error if this higher sample happens to come from the low Gaussian. In the interrogation session, accuracy corresponds to the selection of the alternative which received higher evidence in that trial, as selecting based on this is the best strategy a subject can adopt.

Three measures of confidence resolution were computed: i) the difference between the confidence of correct and error responses, ii) the trial by trial Gamma correlation between confidence and accuracy (Nelson, 1984), iii) the type-2 AUROC measure of metacognitive performance (Fleming \& Lau, 2014). 
Table-1: Average choice accuracy and measures of confidence-resolution

Accuracy Confidence- Gamma correlation Type 2 AUROC

\begin{tabular}{ccccc}
\hline Free response & 0.81 & 0.87 & 0.51 & 0.67 \\
\hline Interrogation & 0.81 & 1.01 & 0.58 & 0.7 \\
\hline \multirow{2}{*}{-test } & $\begin{array}{r}t(16)=0.4, p=.66, \\
\text { Cohen's } d=.1\end{array}$ & $\begin{array}{c}t(16)=2.3, \boldsymbol{p}=. \mathbf{0 3}, \\
\text { Cohen's } d=.56\end{array}$ & $\begin{array}{c}t(16)=1.8, \boldsymbol{p}=. \mathbf{0 8}, \\
\text { Cohen's } d=.44\end{array}$ & $\begin{array}{c}t(16)=2.1, \boldsymbol{p}=. \mathbf{0 5}, \\
\text { Cohen's } d=.51\end{array}$ \\
\hline
\end{tabular}

We observe that choice accuracy is highly similar in the two sessions (note, however, that session two has the advantage of practice, and also the criterion is adjusted to the overall evidence, which is what subjects can judge). In contrast, all three measures of confidence resolution show improved metacognitive performance in the interrogation session. This reaches statistical significance for two of the measures and is marginal for the third one. Finally, in Fig. 4 we examine the correlation between confidence and RT in the two sessions.
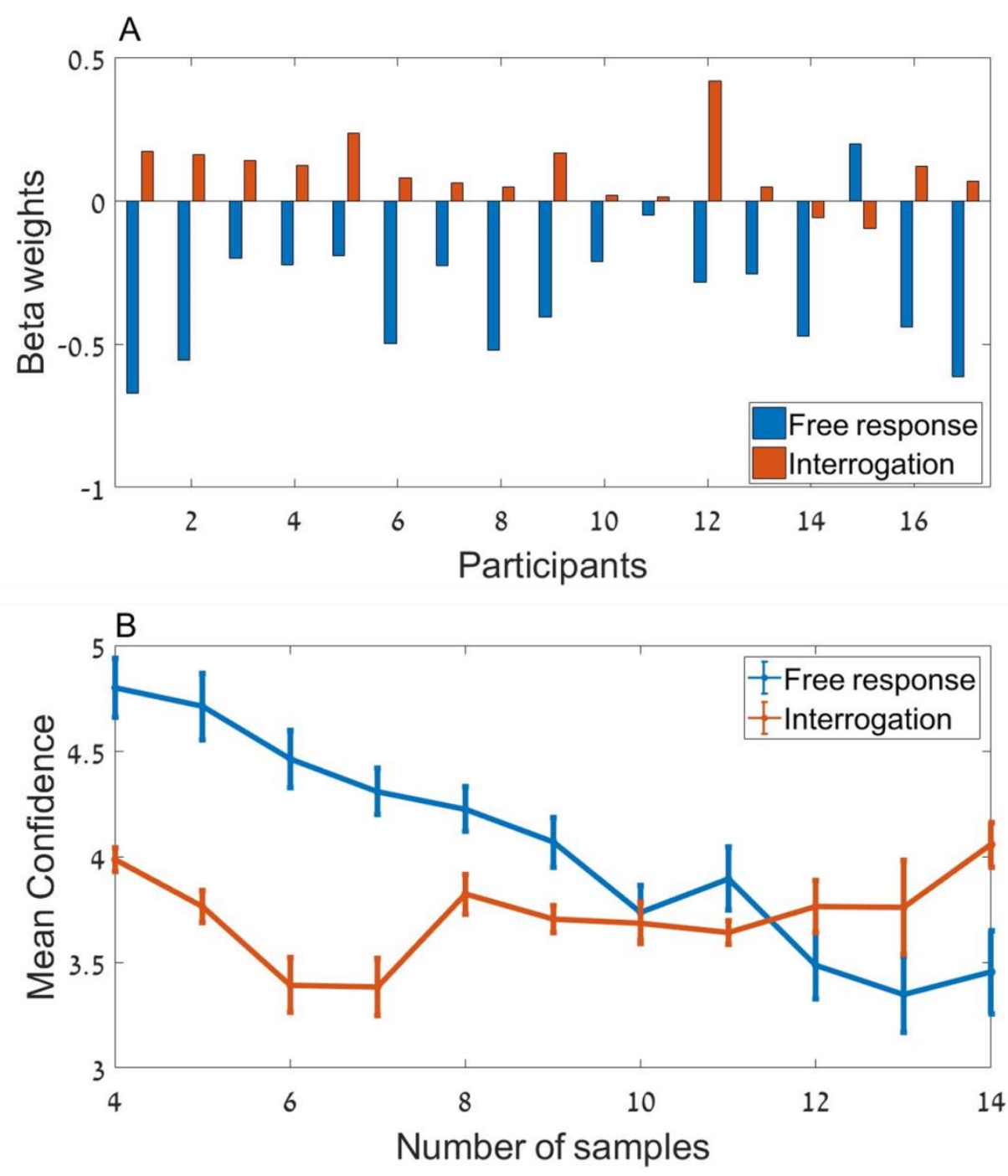
Fig.4. A) Beta weights for the regression RT on confidence for each participant in the free response and the interrogation sessions in Experiment 1. The average coefficient across participants is -.34 (SD=.19) for the free response session, and $0.1(S D=.1)$ for the interrogation session. B) Group mean confidence as a function of $R T$ (number of samples before responding) in the two sessions. The error bars represent within-subjects SE, following Cousineau (2005).

We observe that the typical negative correlation between confidence and RT is only found in the free response task (Mean $\beta=-.34, \mathrm{SD}=.19$ ). This indicates that in free-response situations participants use the number of samples they examined as a proxy for the task difficulty and thus for confidence (Kiani et al., 2014; Zakay \& Tuvia, 1988). Alternatively, subjects could rely on a collapsing boundary to form their choice (Glickman \& Usher, 2019; Malhorta, Leslie et al., 2017; Palestro et al., 2018; Evans et al., 2020), and then use this time as a proxy for the level of the boundary reached at decision time. In the interrogation session, on the other hand, the correlation between confidence and accuracy is positive (mean=.1, $\mathrm{SD}=.1$ ). These dissociations between confidence and RT (as a result of the self-termination vs controlled decision-time) is consistent with previous literature (see benchmark 4 and 5 (Table-2) in Pleskac and Busemeyer (2010) and additional references there). We defer an explanation of this difference for the model analysis section.

Based on these results, we carried out a pre-registered experiment (https://osf.io/gt2qz/) on a larger cohort of participants $(N=35)$. This sample size was selected based on an a priori power analysis that was conducted, which tested the difference between confidence resolution in session 1 and session 2 of Exp. 1 (using a two-tailed paired t-test with an alpha of .01), showing that 35 participants are required to achieve a power of .80 .

\section{Experiment 2}

In Table 2 we report the same measures of choice accuracy and of metacognitive performance as used in analysis of Experiment 1.

Table-2

\begin{tabular}{ccccc}
\hline & Accuracy & $\begin{array}{c}\text { Confidence- } \\
\text { resolution }\end{array}$ & Gamma correlation & Type 2 AUROC \\
\hline Free response & 0.79 & 0.84 & 0.49 & 0.67 \\
\hline Interrogation & 0.83 & 1.1 & 0.59 & 0.72 \\
\hline$t$-test /Ancova & $\begin{array}{c}t(34)=5.7, p<.001, \\
\text { Cohen's } d=.97\end{array}$ & $\begin{array}{c}F(1,33)=2.7, p=.1, \\
\text { Cohen's } d=.36\end{array}$ & $\begin{array}{c}F(1,33)=4.9, p=.03, \\
\text { Cohen's } d=.44\end{array}$ & $\begin{array}{c}F(1,33)=5, p=.03, \\
\text { Cohen's } d=.45\end{array}$ \\
\hline
\end{tabular}


We find that the choice accuracy is significantly higher in the second session (again, this includes benefits from practice). All three measures of metacognition are higher in the interrogation session. In order to establish that such differences are not simply due to differences in task accuracy, we carried out an ANCOVA analysis which compared between the metacognitive performance measures while controlling for differences in accuracy. Note that an alternative approach here would be to use a modelbased estimate of metacognitive efficiency (i.e. metacognitive performance controlling for task performance) such as meta-d'/d' (Maniscalco \& Lau, 2012). However, due to the meta- $d$ ' framework assuming an underlying SDT model for confidence, which we anticipate does not to hold for the freeresponse session, we instead focus on model-free estimates of metacognitive performance and control for task performance differences post-hoc. After controlling for performance, we continued to obtain higher metacognitive performance in the interrogation compared to the free response task, as predicted, with significant differences in two of the measures and a trend in the third one. We note that the type-2 AUROC, which unlike gamma is unaffected by biases in overall confidence level (Fleming \& Lau, 2014) was consistently higher in the interrogation session in both Experiments 1 and 2. Finally, we examined if the improvement in metacognition is correlated with the improvement in choice accuracy. There was no correlation between these measures $(r=-0.02)$, further suggesting that the metacognitive improvement is not due to differences in task performance.

In order to better understand differences between the mechanisms of confidence formation in the free response and interrogation sessions, we first examined the correlation between confidence and RT (in the two sessions). Replicating the findings of Experiment 1, the correlation between confidence and RT is negative only in the free response session but not the interrogation session (Fig. 5). 

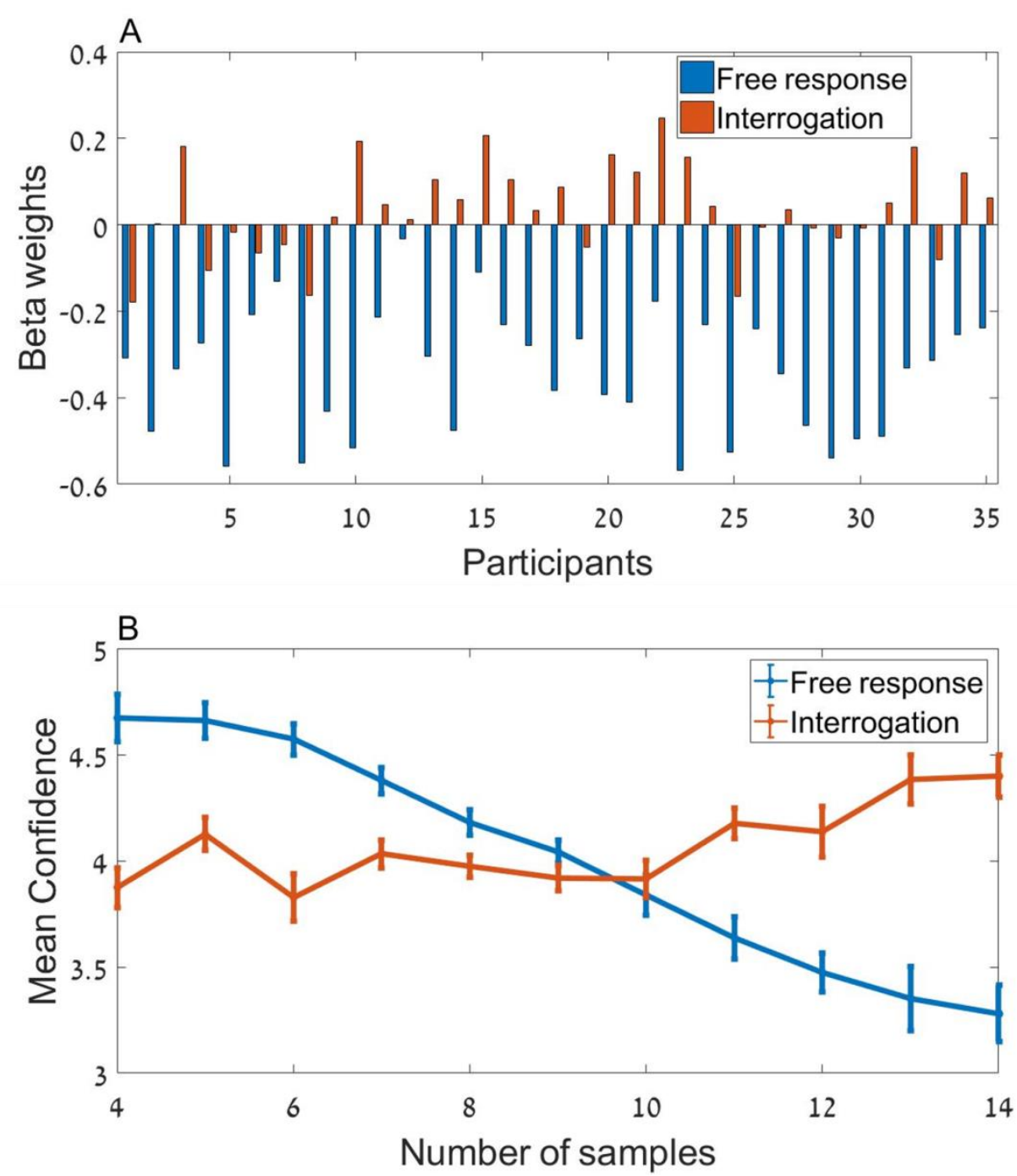

Fig. 5 A) Beta coefficients of the effect of $R T$ on confidence for each participant in the free response and the interrogation session in Experiment 2. The average coefficient over participants is -.34 (SD=.14) for the free response session, and $0.03(S D=1)$ for the interrogation session. B) Group mean confidence as a function of $R T$ (number of samples prior to reaching a decision) in the two sessions. Error bars represent within-subjects SE (Cousineau ,2005).

Second, as indicated by the fits of alternative computational models (see Supplement for details), we find that in the free response sessions, participants' choices are best described by an integration to a collapsing boundary model (Baldson, Wyart, Mamassian 2020; Glickman \& Usher, 2019; Malhorta, Leslie Ludwig \& Bogacz, 2017; Palestro et al., 2018; Evans, Hawkins \& Brown, 2020), which is optimal under conditions that mix difficulty levels (Malhorta et al., 2017). In turn, confidence in the free response session was best accounted by three predictors: i) the evidence from the last item in the sequence, ii) the remainder of the evidence and iii) RT (consistent with a robust negative correlation observed between confidence and RT; Fig. 4-5). In the interrogation session, on the other hand, we find that choices are well accounted by the signal detection theory (SDT) model based on integrated 
evidence (see also Fig. S5 in Supplement, for reverse correlation analysis ruling out an implicit boundary for evidence integration; Kiani et al., 2008). For confidence, we find that the best predictors are the total accumulated evidence or a leaky-integration of the evidence with an average leak factor of 0.88 per frame (i.e., .88 of the integrated-evidence is maintained at every time frame while the rest leaks; Teodorescu \& Usher, 2013; see Supplement for Model description and parameters). ${ }^{3}$ Importantly, as illustrated in Fig. 4-5, unlike in the free-response task, the trial length (number of frames) is now positively correlated with confidence. Further analysis indicates that this is due to the association between time and amount of evidence in this task: the correlation becomes null once the total evidence is partialed out (Fig. S4, Suppl.)

\section{Exp. 1-2 Discussion}

In the first two experiments we found support for the idea that the decision strategy adopted (free response or interrogation) affects metacognitive performance. In particular, when participants are allowed to freely choose when to respond (i.e., when they integrate to an internal decision boundary) their metacognitive performance is lower than when they decide based on an exogenously determined number of samples. However, it can be argued that these results may be caused by a confound related to how accuracy is being measured in the two tasks. In the free-response task, a correct choice was defined according to the generating distributions (the nominal evidence) - which is how the task was presented to participants (they needed to decide which evidence stream, left or right, corresponded to the higher payoff distribution; see Fig 2). In contrast, in the interrogation task the correct choice was defined by the actual stream that was presented to the subject (the actual evidence). Note that in the interrogation condition, deciding about the actual evidence is more natural to the subjects, since they have no way to obtain additional evidence to dissociate between actual accuracy (what they see) and nominal accuracy (i.e., the generating distribution). Moreover, in a fraction of "conflict" trials (on average, $13 \%$ for the interrogation session and 5\% for the free-response session), these two accuracy criteria (actual/nominal) differ. For example, one may have a trial in which the higher generating evidence stream is on the Left, but at the time of response, the Right sequence had delivered a higher amount of observed evidence. The fraction of such conflict trials is higher in the interrogation session, since in this session, the evidence is sampled afresh independent of the number of evidence samples. By contrast in the free-response task, there is a dependency between the evidence and the number of samples, so that short evidence trials are the ones which were easier (resulting in faster integration to

\footnotetext{
3 The leaky-integration achieves a higher log-likelihood but the total (non-leaky) evidence in favored in terms of model
} complexity (BIC) measures. 
boundary). Consequently, if the subject responds in such a conflict trial based on the actual evidence, this counts as a mistake in the free response task (where the task was to decide on the generating distribution and it was the subject's fault for deciding too soon), but counts as a correct response in the interrogation session (where the subjects did not have any way to determine that the actual evidence is contrary to the nominal evidence). While this is consistent with the task instructions, it may lead, in principle, to a performance bias favoring the interrogation session, and thus could provide an explanation for the improved metacognitive performance in this condition.

One way to partially address this concern would be to use (contrary to task instructions) the same criterion of 'correctness' in the two tasks when analyzing metacognitive performance. We re-analyzed the results of Exp-2 using the 'actual' evidence criterion (the accuracy defined by what subjects had observed prior to responding) in both sessions. While the subjects were instructed to decide based on nominal evidence in the free response session, one may reason that if a subject responded too fast and encountered evidence that was in conflict with the generating distribution, the subject may still judge confidence based on the actual evidence (this is not strictly correct and we address it in Exp-3, see below). When we score metacognitive performance based on this actual evidence criterion in both the free response and interrogation sessions, we still find that the metacognitive performance remains significantly higher in the interrogation session (Type 2 AUROC for free response $=0.68$, and for interrogation $=0.72, t(34)=2.13, p=.039)$.

A better way to address the accuracy criterion concern is to score performance based on 'nominal' criteria in both sessions. This is because we aimed for the interrogation session to be a 'simulation' of the performance that would be achieved in free-response task in which the participants used no integration to determine the length of evidence (e.g., they deployed a 'random-timer' strategy; Glickman \& Usher, 2019). Thus, scoring both sessions based on nominal accuracy criteria is more consistent with this rationale. The problem with using nominal criteria in both sessions is that in the interrogation session there was a higher fraction of conflict trials (short trials in which the actual evidence went against the generating distributions) and the participants were told to respond based on the evidence they see at the end of the sequence. To deal with this, we carried out a third experiment, which presented the instructions based on nominal criteria in both sessions ${ }^{4}$ and in which we also aimed to reduce the fraction of conflict trials by design.

4 This cannot make a difference for choice, but can do so for confidence. For example, consider a trial that presents the following 2-frame evidence sequence: F1 $(7,7)$; F2 $(8,7)$. Even though it is clear that the total evidence favors the left 


\section{Experiment 3}

In this experiment we emphasized nominal choice accuracy (i.e. the requirement to indicate the higher generating distribution) for both choice and confidence in both sessions and we attempted to minimize the fraction of conflict trials (where nominal and actual accuracy conflicted) that subjects encountered in the interrogation session ( $\sim 13 \%$ in Exp. 1-2). To do this, instead of equalizing the number of samples in the two sessions trial by trial, as in Exp. 1-2, in Exp. 3 the number of samples were equalized for each subject on average, across trials in a session. For example, if a subject made, in the first freeresponse session, decisions that integrated between 3-12 evidence frames with an average of 7 frames, in the second interrogation session, the subject received 7 frames evidence on all trials (with evidence streams being newly sampled from the same generating distributions as in the free-response session). This reduces the fraction of ambiguous conflict trials in the second session (which are typically the very short trials), for which the subject has little resources to distinguish between actual and nominal accuracy criteria. Thus this procedure allows us to compare between the integration to boundary strategy, which the subject can deploy in the first session, and the fixed number of samples strategy required in the second session, when task performance is calculated using the same, nominal, criterion for accuracy in both cases. Based on computational considerations, we predict that even under these carefully matched conditions, choice accuracy and metacognitive performance will continue to show a tradeoff - choice accuracy will be higher in the free-response session (as predicted by the SPRT theory; Wald, 1948), while metacognitive performance will be higher in the interrogation session. In order to increase the generality of the task, we also modified the stimuli from sequences of numbers to sequences of lines (Vickers, Burt, Smith \& Brown, 1985)

\section{Methods}

Participants. 50 undergraduates from Tel-Aviv University (34 females; age: $\mathrm{M}=23$, range 21-28 years) participated in the experiment. The participants received course credit in exchange for taking part in the experiment. The experiment was approved by the ethics committee at TAU. The sample size was selected based on a power analysis for the confidence resolution effect in Experiment 2, showing that 50 participants are required to achieve a power of $90 \%$ for alpha of .05 .

Procedure and design. The task was similar to the previous experiments task with a few exceptions: I) alteration in how stimulus evidence was generated, as described in detail above, ii) Instead of numbers, alternative, one can understand that the likelihood that the generating distribution favors the left response is not high, and this could be reflected in confidence judgments. 
participants were presented with sequences of vertical lines with different lengths (pairs of vertical lines, with lengths sampled from the same overlapping Gaussians). iii) The sequence rate was changed to $0.6 \mathrm{~s}$ per item (the $0.6 \mathrm{~s}$ consisted of $0.3 \mathrm{~s}$ where the stimuli were presented and $0.3 \mathrm{~s}$ of a blank screen in order to separate distinct presentations of the bar stimuli). iiii) Each session consisted of 2 blocks with 40 trials each. The rest of the procedure was identical to the previous experiments.

\section{Results}

In Table 3 we report measures of choice accuracy and metacognitive performance, all based on nominal evidence.

Table-3

Accuracy Confidence- Gamma correlation Type 2 AUROC
resolution

\begin{tabular}{ccccc}
\hline Free response & 0.85 & 1 & 0.53 & 0.69 \\
\hline Interrogation & 0.82 & 1.2 & .62 & 0.72 \\
\hline \multirow{2}{*}{-test } & $\begin{array}{c}t(49)=3.5, \boldsymbol{p}<. \mathbf{0 0 1}, \\
\text { Cohen's } d=.5\end{array}$ & $\begin{array}{c}t(49)=1.9, \boldsymbol{p}=. \mathbf{0 6}, \\
\text { Cohen's } d=.27\end{array}$ & $\begin{array}{c}t(49)=2.2, \boldsymbol{p}=. \mathbf{0 2 9}, \\
\text { Cohen's } d=.31\end{array}$ & $\begin{array}{c}t(49)=2.2, \boldsymbol{p}=. \mathbf{0 2 9}, \\
\text { Cohen's } d=.31\end{array}$ \\
\hline
\end{tabular}

As predicted, we find that the choice accuracy is significantly higher in the free response session (as expected from measuring the nominal distributions in both tasks). In contrast, and again in line with our predictions, the confidence resolution in all three measures of metacognition is higher in the interrogation session compared to the free response session, with significant differences in two of the measures and a trend in the third one (see Fig. 6). Notably, the bias-free type 2 AUROC metric of metacognitive sensitivity revealed significantly increased confidence resolution in the interrogation condition despite first-order performance being lower in this condition. Seeing as choice accuracy is itself known to positively affect type 2 AUROC estimates (Fleming \& Lau, 2014), this double dissociation is very strong evidence of a metacognitive advantage in the interrogation condition. 


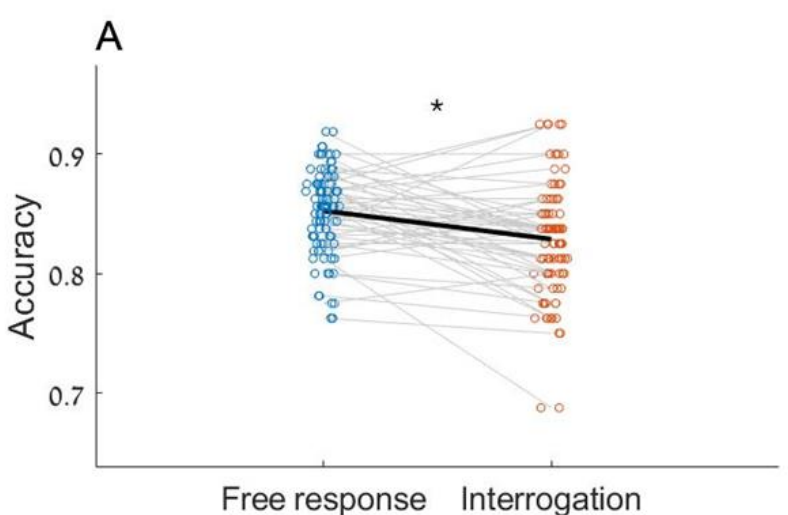

Free response Interrogation

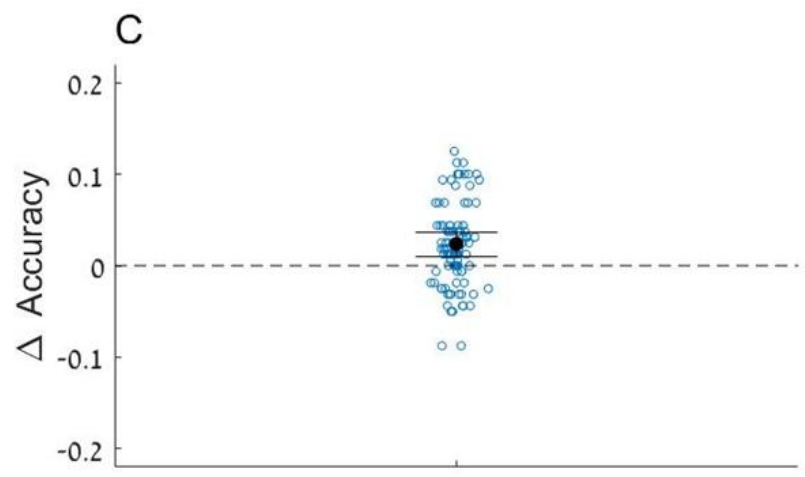

(Free response-Interrogation)

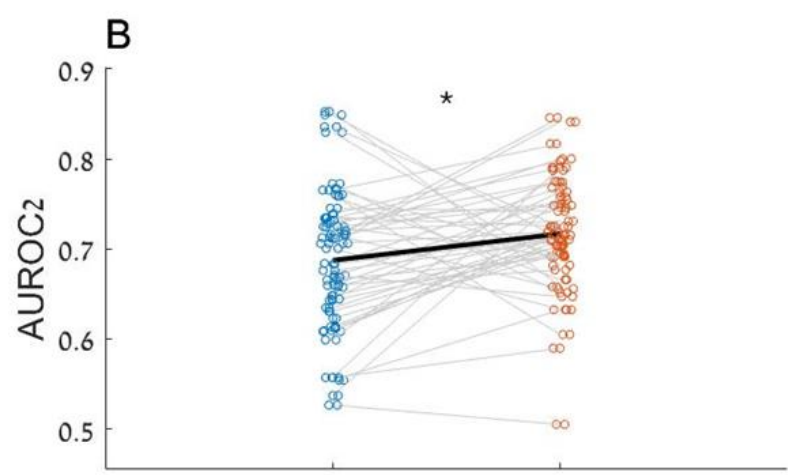

Free response Interrogation

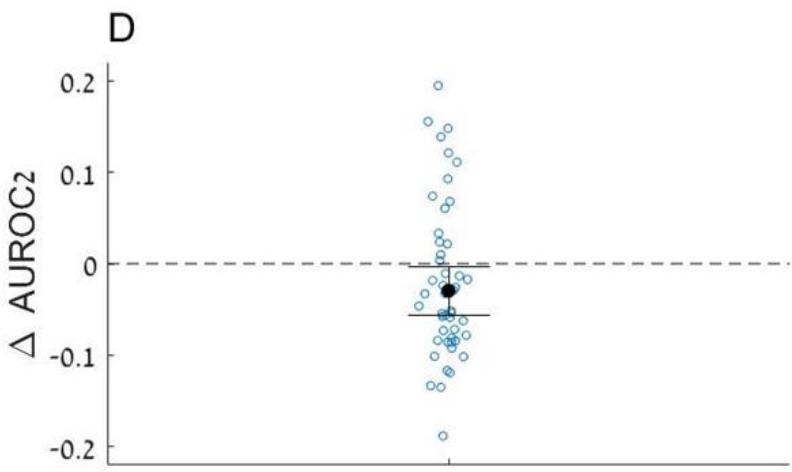

(Free response-Interrogation)

Fig. 6. Experiment 3 results: A double dissociation in choice accuracy and metacognitive performance (quantified as AUROC2) between the free response (blue) and interrogation (red) sessions. While firstorder choice accuracy (panel A) was higher in the free response session, metacognitive performance (panel B) was higher in the interrogation session. Blue and red circles correspond to the performance measure (accuracy in panel A\& AUROC2 in panel B) of each participant in the FR and interrogation sessions, respectively. Black line corresponds to the average score of each session. C) The difference in accuracy for each participant between the free response and interrogation sessions. D) The difference in AUROC2 for each participant between the free response and interrogation sessions. Error bars correspond to $95 \%$ confidence interval. Black dot corresponds to the mean score. ${ }^{*} p<.05$.

\section{General Discussion}

Across three experiments we found that the integration to boundary strategy, which allows participants to make endogenous decisions on streams of evidence, has a cost in metacognitive performance relative to a fixed number of samples strategy in which the evidence is exogenously presented to the subject. Critically, this is the case even when the amount of evidence presented in the two cases is identical (either matched trial by trial in Exp. 1-2 or on average, across trials, in Exp-3), and when any 
performance differences between the two task variants are controlled for (no accuracy difference in Exp-1, and ANCOVA in Exp-2). Moreover, in Exp. 3, we obtained a striking double dissociation between cognitive and metacognitive performance (Fig. 6). As predicted by theoretical models of evidence accumulation, the integration to boundary strategy results in a higher choice accuracy but in lower metacognitive performance when the number of samples is the same, on average, to an equivalent evidence stream that cannot be interrupted. This double dissociation rules out accounts of these findings in terms of practice effects (as choice accuracy is lower in session 2 than session 1), or in terms of first-order performance itself affecting metrics of metacognition - as the change in metacognition is opposite in sign to the change in first-order performance. This result also provides support for the idea that while integration to boundary is optimal for maximizing choice accuracy, it is less optimal for metacognition, as it leaves less variability in evidence (that was not already used in the decision) to signal confidence.

One limitation of the study is that the interrogation session was always presented second. This procedure was devised because we wanted to equalize the amount of evidence that subjects sampled to the free response session, and since the latter is under the subject's control, this had to be measured first. However, this procedure could, potentially, result in a practice confound - the improved metacognitive performance in the interrogation session could be due to increased familiarity with the task. While this improvement was only consistently observed in metacognitive, and not in choice, performance, one could reason that the latter is masked by the fact that choice performance is predicted to be lower in the interrogation protocol (Wald, 1948). We believe, however, that such a practice account is unlikely for our results. First, our participants received no (trial by trial) accuracy feedback, so they had little input to guide them in calibrating their confidence responses to objective reality (Carpenter et al., 2019). Second, we carried out two additional analyses to rule out explanations in terms of a practice confound. In a first analysis, we examined metacognitive performance in Exp-3 in the first vs second half of each session. This analysis did not reveal any significant effect of sessionhalf (first half, mean type 2 AUROC $=0.66$, second half; mean type 2 AUROC $=0.67 ; t(49)=.4, \boldsymbol{p}=.68$ ). One may still argue that the practice needed to obtain improvement in our task requires a full session to emerge (80 rather than 40 trials). In order to rule out this possibility we ran a control version of our experiment-3 (N=35), in which participants completed two consecutive blocks of the free response task, each of 80 trials; see control experiment methods in Suppl for details). As expected (based on lack of accuracy feedback) there was no improvement in metacognitive performance between blocks (first 
block, mean type 2 AUROC $=0.66$; second block, mean type 2 AUROC $=0.67 ; t(34)=.49, \boldsymbol{p}=.62)$. Together with the double dissociation between choice and metacognitive performance observed in Exp 3 (Fig, 6), the results of this control experiment make it unlikely that a practice effect could account for our key findings. Rather we believe that these additional analyses support our conclusion that integration to bound is beneficial for choice performance, but has a cost in metacognitive performance.

Interestingly, previous studies of perceptual metacognition often rely on tasks in which the number of evidence samples is exogenously determined, allowing for an enhanced confidence resolution. In addition, model-based measures of metacognitive efficiency such as meta- $d$ ' implicitly assume an underlying SDT generative model of confidence, which may only be suitable for interrogation-like cases, but not for more naturalistic free-response settings where the decision to terminate information search is under the subject's control (Yeung \& Summerfield, 2012). Future work should contrast these two decision strategies - integration-to-boundary and deciding from a fix number of samples - in tasks such as wagering (Persaud, McLeod \& Cowey, 2007), which combine choice and confidence into a unified performance measure, and explore alternative model-based metrics of metacognitive performance that incorporate decision time.

In addition to these differences in metacognitive performance, we also found that the way in which participants generate their choice and confidence estimates differed in the two experimental sessions. In the free-response task, the best-fitting choice model utilisted integration to a collapsing boundary, while the confidence was based on both RT (which for this model is associated with the boundary level at response) and the last piece of evidence, which is likely to involve post-decision integration (Pleskac \& Busemeyer, 2010; Moran et al, 2015). Both of these factors could contribute to a non-zero level of confidence resolution in the free-response task. Indeed, as RTs tend to be faster for correct (compared with incorrect) responses (Fig. S3, Supplement), RT itself provides distinctive information on choice accuracy. An impact of post-decisional evidence integration on confidence resolution in the freeresponse task is also supported by a median split of the confidence-resolution between trials in which the response was entered before or after the median RT on the last frame before stimulus termination (Fig. 7). We reasoned that for faster RTs (measured from the last presented frame) there is a higher chance that the decision was based on evidence presented before the last frame was presented and thus this extra frame could serve as post-decision evidence. Consistent with post-integration models 
(Pleskac \& Busemeyer, 2010), we find that metacognitive performance (AUROC2) is higher when such post-decision evidence is available $(t(34)=2.12 ; p<0.05)$.
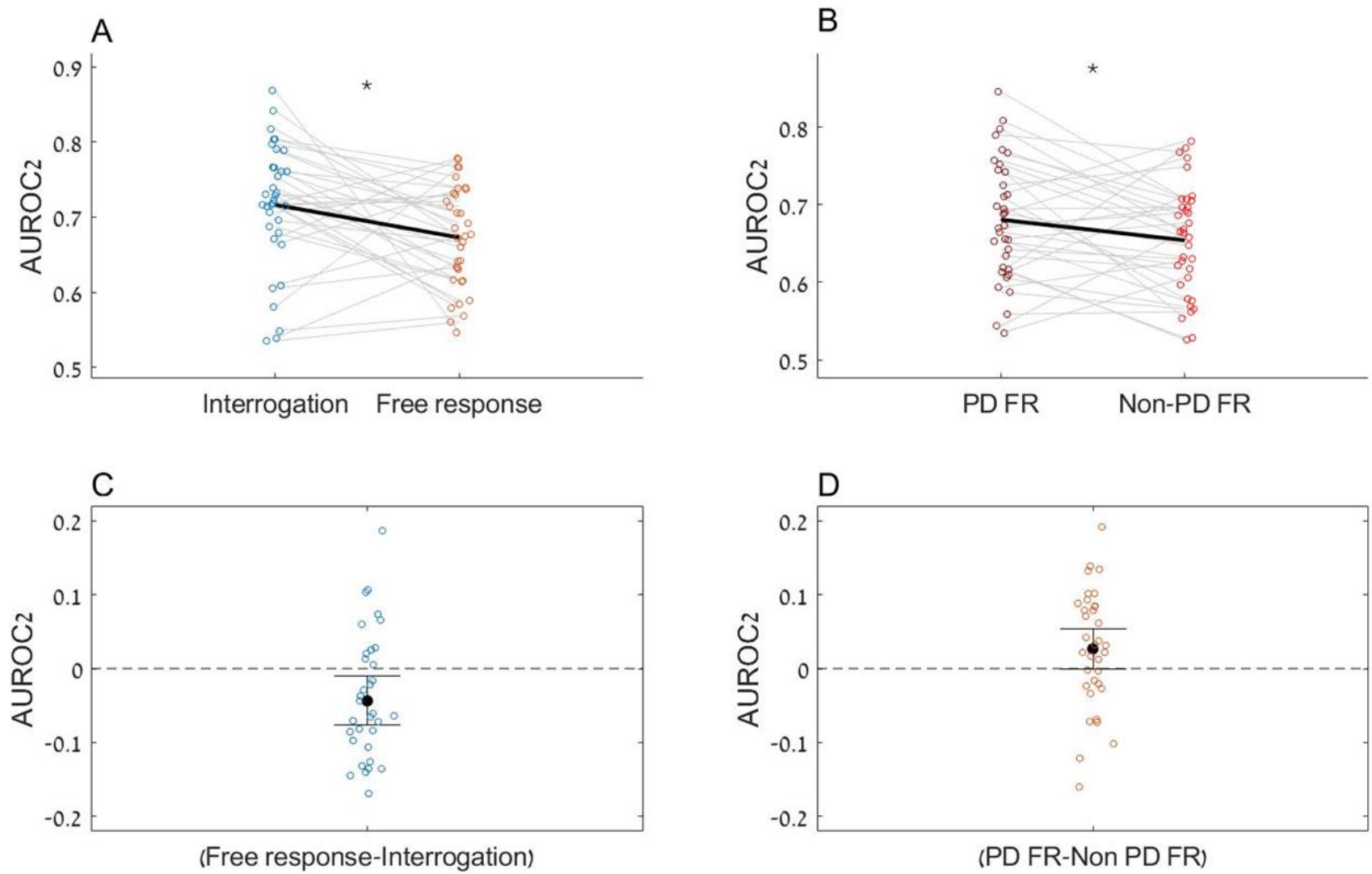

Fig. 7. A) Metacognitive performance (type 2 AUROC measure), in the interrogation (blue) and free response (FR-red) sessions (experiment 2). B) The FR session was further separated into fast (dark red) and slow (light red) trials based on a median split of RT to the final sample, as a proxy for a contribution of post-decision evidence to confidence (PD=post decision). C) The difference in AUROC2 for each participant between the free response and interrogation sessions. D) The difference in AUROC2 for each participant between the fast (PD) and slow (non-PD) trials in the free-response session. Error bars correspond to 95\% confidence interval. Black dot corresponds to the mean score.

In the interrogation session, on the other hand, we find that choices are well accounted for by a signaldetection model applied to the whole evidence stream. For confidence, we find that the best predictors are simply the total accumulated evidence or a leaky-integration of the evidence. This supports the signal-detection model of confidence (see Fig. 1 and 3B), according to which, when evidence is exogenously presented (as in interrogation), the same information - the integrated evidence - is used to guide both choices and for confidence. This is also consistent with the fact that in the interrogation condition, the trial length (number of frames) is positively correlated with confidence. Further analysis 
indicates that this is due to the association between time and amount of evidence in this task: the correlation between confidence and time becomes null once the total evidence is partialed out (Fig. S4, Suppl.)

To conclude, while a reliance on RT and on post-decision integration allows participants to obtain positive confidence resolution in the free-response task (following integration to a collapsing boundary), this does not facilitate the same level of metacognitive performance as that obtained when basing confidence on the total evidence encountered, as is possible in the interrogation task (see also Fig. S2; Supplement). Instead, our results indicate that participants adapt the confidence mechanism they deploy depending on the task contingencies, and adopt different strategies to extract some form of metacognitive performance even in an integration-to-bound setting where confidence resolution is likely to be compromised. In particular, they rely on total evidence when the termination of the evidence stream is independent of their choice, as normatively supported by signal detection models. However, when faced with a self-terminated task, participants use integration to a collapsing boundary to provide an efficient compromise between optimizing choice accuracy (Tajima, Drugowitsch, \& Pouget, 2016; Malhorta et al., 2017) and confidence resolution (see Fig S3 in Suppl). In this setting, they can also rely on cues from both RT and post-decision evidence to further boost their metacognitive performance (see Fig S6 in Supplement for a simulation showing that the collapsing boundary model provides the most efficient balance of choice accuracy and metacognition in the context of a wagering task, where confidence resolution also contributes to the magnitude of reward received (Persaud, McLeod \& Cowey, 2007)). How such additional cues to performance are integrated with the available stimulus evidence remains a topic for future work. One possibility is that higher-order mechanisms supporting metacognition have access to parallel streams of information, including one's own actions and response time, allowing more efficient inference on self-performance (Fleming \& Daw, 2017).

\section{Conclusions}

The fidelity of metacognition is instrumental to enhancing performance in a variety of contexts, such as when confidence is being used to guide subsequent decisions (Persaud, McLeod \& Cowey, 2007; van den Berg et al., 2016) or when deciding whether or not to seek new information (Schulz et al., 2021). For example, in an exam context, subjects can use feelings of confidence to decide whether or not their work is ready to be submitted for grading, or whether to continue working on it. Crucially, such 
decisions require a high degree of metacognitive competence (confidence resolution) - we would not want to have high feelings of confidence in the context of low accuracy. Previous studies have focused on how metacognitive competence is affected by various factors such as task domain and mental health, and sought to unveil the neural correlates of confidence formation (Hoven et al., 2019). However, the impact of different strategies for decision-making - that might vary, within the same individual, from context to context - on metacognition have remained unexplored. Here, by using carefully controlled evidence accumulation paradigms informed by computational modeling, we find that i) a strategy of integrating to a fixed boundary, while theoretically optimal for choice accuracy, has a cost in confidence resolution and that ii) participants sought to adapt the way that they formed their confidence in such settings in order to retain some degree of metacognitive competence (see Fig. 4-5 and Model selection in Suppl.). Interestingly, a strategy of integrating to a collapsing boundary appears to be a good compromise for optimizing both choice accuracy and metacognition. These results further imply that human observers meta-optimise metacognition itself - altering how confidence is formed depending on the task context at hand. The mechanisms of such metacognitive optimization remain a rich topic for future investigation.

\section{References}

Bahrami, B., Olsen, K., Latham, P. E., Roepstorff, A., Rees, G. \& Frith, C. D. (2010). Optimally interacting minds. Science, 329, 1081-1085. doi:10.1126/science.1185718

Balsdon, T., Wyart, V. \& Mamassian, P. (2020). Confidence controls perceptual evidence accumulation. Nature Communications, 11 (1). doi:10.1038/s41467-020-15561-w

Bogacz, R., Brown, E., Moehlis, J., Holmes, P. \& Cohen, J. D. (2006). The physics of optimal decision making: A formal analysis of models of performance in two-alternative forced-choice tasks. Psychological Review, 113 (4), 700-765. doi:10.1037/0033-295X.113.4.700

Calder-Travis, J., Charles, L., Bogacz, R., \& Yeung, N. (2020). Bayesian confidence in optimal decisions. psyArXiv.

Carlebach, N. \& Yeung, N. (2020). Subjective confidence acts as an internal cost-benefit factor when 1175 choosing between tasks. Journal of Experimental Psychology: Human Perception and Performance. 1176 doi:10.1037/xhp0000747

Carpenter, J., Sherman, M. T., Kievit, R. A., Seth, A. K., Lau, H., \& Fleming, S. M. (2019). Domaingeneral enhancements of metacognitive ability through adaptive training. Journal of Experimental Psychology: General, 148(1), 51. 
Cousineau, D. (2005). Confidence intervals in within-subject designs: A simpler solution to Loftus and Masson's method. Tutorials in quantitative methods for psychology, 1(1), 42-45.

De Martino, B., Fleming, S. M., Garrett, N. \& Dolan, R. J. (2013). Confidence in value-based choice. Nature Neuroscience, 16, 105-110. doi:10.1038/nn.3279

Evans, N. J., Hawkins, G. E., \& Brown, S. D. (2020). The role of passing time in decisionmaking. Journal of experimental psychology: learning, memory, and cognition, 46(2), 316.

Fleming, S. M., \& Lau, H. C. (2014). How to measure metacognition. Frontiers in human neuroscience, 8,443 .

Glickman, M., \& Usher, M. (2019). Integration to boundary in decisions between numerical sequences. Cognition, 193, 104022.

Hainguerlot, M., Vergnaud, J. \& de Gardelle, V. (2018). Metacognitive ability predicts learning cuestimulus associations in the absence of external feedback. Sci Rep 8, 5602.

Hoven, M., Lebreton, M., Engelmann, J. B., Denys, D., Luigjes, J., \& van Holst, R. J. (2019). Abnormalities of confidence in psychiatry: an overview and future perspectives. Translational psychiatry, 9(1), 1-18.

Kepecs, A., \& Mainen, Z. F. (2012). A computational framework for the study of confidence in humans and animals. Philosophical Transactions of the Royal Society B: Biological Sciences, 367(1594), 1322-1337.

Kiani, R., Corthell, L. \& Shadlen, M. N. (2014). Choice certainty is informed by both evidence and decision time. Neuron, 84 (6), 1329-1342. doi:10.1016/j.neuron.2014.12.015

Kiani, R., Hanks, T. D., \& Shadlen, M. N. (2008). Bounded integration in parietal cortex underlies decisions even when viewing duration is dictated by the environment. Journal of Neuroscience, 28(12), 3017-3029.

Malhotra, G., Leslie, D. S., Ludwig, C. J., \& Bogacz, R. (2017). Overcoming indecision by changing the decision boundary. Journal of Experimental Psychology: General, 146(6), 776.

Maniscalco, B., \& Lau, H. (2012). A signal detection theoretic approach for estimating metacognitive sensitivity from confidence ratings. Consciousness and cognition, 21(1), 422-430.

Merkle, E. C., \& Van Zandt, T. (2006). An application of the Poisson race model to confidence calibration. Journal of Experimental Psychology: General, 135(3), 391-408.

Moran, R., Teodorescu, A. R., \& Usher, M. (2015). Post choice information integration as a causal determinant of confidence: Novel data and a computational account. Cognitive psychology, 78, 99147. 
Palestro, J. J., Weichart, E., Sederberg, P. B., \& Turner, B. M. (2018). Some task demands induce collapsing bounds: Evidence from a behavioral analysis. Psychonomic bulletin \& review, 25(4), 1225-1248.

Pleskac, T. J. \& Busemeyer, J. R. (2010). Two-stage dynamic signal detection: A theory of choice, 1307 decision time, and confidence. Psychological Review, 117 (3), 864-901. doi:10.1037/a0019737 1308

Persaud, N., McLeod, P. \& Cowey, A. (2007). Post-decision wagering objectively measures awareness. Nat Neurosci 10, 257-261. https://doi.org/10.1038/nn1840

Rahnev, D. \& Denison, R. N. (2018). Suboptimality in perceptual decision making. Behavioral and Brain 1309 Sciences, 41. doi:10.1017/S0140525X18000936

Ratcliff, R., Smith, P. L., Brown, S. D. \& McKoon, G. (2016). Diffusion decision model: Current issues and history. Trends in Cognitive Sciences, 20 (4), 260-281. doi:10.1016/j.tics.2016.01.007

Ratcliff, R., \& Starns, J. J. (2009). Modeling confidence and response time in recognition memory. Psychological review, 116(1), 59-83. https://doi.org/10.1037/a0014086

Reynolds, A., Kvam, P. D., Osth, A. F., \& Heathcote, A. (2020). Correlated racing evidence accumulator models. Journal of Mathematical Psychology, 96, 102331.

Rollwage, M., Loosen, A., Hauser, T. U., Moran, R., Dolan, R. J., \& Fleming, S. M. (2020). Confidence drives a neural confirmation bias. Nature communications, 11(1), 1-11.

Sanders, J. I., Hangya, B. \& Kepecs, A. (2016). Signatures of a statistical computation in the human 1331 sense of confidence. Neuron, 90 (3), 499-506. doi:10.1016/j.neuron.2016.03.025

Schulz, L., Fleming, S. M., \& Dayan, P. (2021). Metacognitive Computations for Information Search: Confidence in Control. bioRxiv.

Tajima, S., Drugowitsch, J., \& Pouget, A. (2016). Optimal policy for value-based decision-making. Nature communications, 7(1), 1-12.

Teodorescu, A. R., \& Usher, M. (2013). Disentangling decision models: From independence to competition. Psychological review, 120(1), 1.

van den Berg, R., Anandalingam, K., Zylberberg, A., Kiani, R., Shadlen, M. N. \& Wolpert, D. M. (2016). A common mechanism underlies changes of mind about decisions and confidence. eLife, 5, 1-21. 1353 doi:10.7554/eLife.12192

Vickers, D. (1979). Decision processes in visual perception. Academic Press series in cognition and perception. New York ; London: Academic Press

Vickers, D., Smith, P., Burt, J., \& Brown, M. (1985). Experimental paradigms emphasising state or 
process limitations: II effects on confidence. Acta Psychologica, 59(2), 163-193.

Wald, A. \& Wolfowitz, J. (1948). Optimum character of the sequential probability ratio test. The Annals of Mathematical Statistics, 19 (3), 326-339. doi:10.1214/aoms/1177730197

Yeung, N. \& Summerfield, C. (2014). Shared mechanisms for confidence judgements and error detection in human decision making. In S. M. Fleming \& C. D. Frith (Eds.), The cognitive neuroscience of metacognition (pp. 147-167). Berlin, Heidelberg: Springer. doi:10.1007/978-3-642-45190-4 7

Zakay, D., \& Tuvia, R. (1998). Choice latency times as determinants of post-decisional confidence. Acta Psychologica, 98(1), 103-115.

\section{Supplement}

\section{Predictions of confidence-resolution}

Here we show simulations for confidence based on: i) integration to boundary + post-decision evidence, ii) vs Signal-Detection for total evidence when the number of samples is fixed. The algorithm of integration to boundary was simulated by sampling pairs of values from two normal distributions: Left $\sim N\left(52,15^{2}\right)$ and Right $\sim N\left(48,15^{2}\right)$, and accumulating the differences of each pair $\left(\sum_{i=1}^{N} L i-R i\right)$. Two types of decision mechanisms were contrasted: i) Free-response/integration to a fixed boundary, in which trials were terminated when the accumulated evidence reached a predefined decision boundary, and ii) Interrogation, in which decision was made after a fixed number of samples. The fixed boundary was set to 43 to equalize the mean accuracy of both decision mechanisms (accuracy $=0.72$ ). The mean number of samples was 6.6 for the Free-response protocol, and was set to 10 for the interrogation protocol 10. In both simulations evidence values higher than the .995 quantiles or lower than the .005 quantiles were considered outliers and excluded than the analyses. Including these values did not change the results.

Free Response. Confidence for the free-response decision mechanism was determined following the 2DSD mechanism (Pleskac \& Busemeyer, 2010, see Fig. S1A). For each simulated trial an extra sample was drawn from the Left \& Right distributions. Confidence was computed based on the consistency between the extra-sample and choice. For example, if a participant chose the left alternative and the extra-sample had a high positive value (i.e., the left number was higher than the 
right one), then the confidence level would be high. As opposed to that, if the extra-sample had a high negative value (i.e., the left number is lower than the right number), the confidence level would be low. Specifically, we calculated the normalized confidence using the following formula:

$$
\text { Normlizedconfidence } \text { Freeresponse }_{\text {Fre }}=\left\{\begin{array}{l}
\frac{E S-\min (E S)}{\max (E S)-\min (E S)}, \text { Left choices } \\
\frac{(E S-\max (E S))}{\max (E S)-\min (E S)}, \text { Right choices }
\end{array}\right.
$$

where $\mathrm{ES}$ is the value of the extra-sample (i.e., $\mathrm{L}_{\mathrm{ES}}-\mathrm{Y}_{\mathrm{ES}}$ ), and $\max (\mathrm{ES}) / \min (\mathrm{ES})$ are the maximum/minimum values of the extra-samples across all trials.

Interrogation. Confidence for the interrogation protocol was determined based on the SDT, using the value of the integrated evidence at the moment of response (see Fig. S1B). The $\mathrm{H}_{1}$ distribution corresponds to the case in which $\mu_{\text {Left }}>\mu_{\text {Right }}$ (i.e., the correct response is left), and the $\mathrm{H}_{2}$ distribution corresponds to the case in which the $\mu_{\text {Left }}<\mu_{\text {Right }}$ (i.e., the correct response is right). Without loss of generality, we consider here only the case in which $t \mu_{\text {Left }}>\mu_{\text {Right }}$, as the other case is symmetrical. For each trial, Confidence was calculated as the absolute value of the difference between the integrated evidence at the moment of response of the criterion (i.e., 0 ). These values were normalized as follows:

$$
\text { Normlizedconfidence }_{\text {Interrogation }}=\frac{|A C-\min (A C)|}{\max (A C)}
$$

where $\mathrm{AC}$ is the value of the accumulated evidence at the moment of response $\left(\sum_{i=1}^{N} L i-R i\right)$, and $\max (\mathrm{AC}) / \mathrm{min}(\mathrm{AC})$ are the maximum/minimum values of the integrated evidence at the moment of response across all trials.

(A)

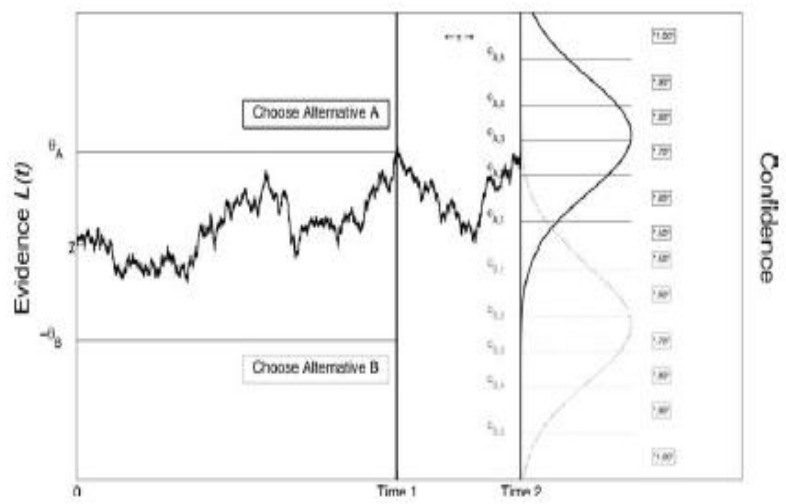

(B)

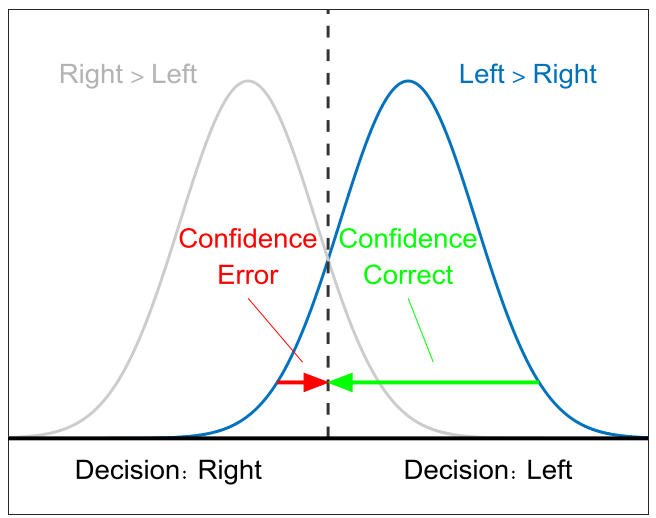


Fig. S1. Confidence resolution in the Free-response and interrogation protocols. (A) Confidence for the free-response protocol was determined by drawing an extra-sample and computing its consistency with choice following the 2DSD mechanism (Pleskac \& Busemeyer, 2010). (B) Confidence was for the interrogation protocol was determined based on the SDT framework by computing the absolute distance of the accumulated evidence at the moment of decision from the criterion. Note that in Fig. 3 in the main text we only present the $\mathrm{H}_{1}$ distribution $\left(\mu_{\text {Left }}<\mu_{\text {Right }}\right)$, as the results are symmetrical for the $\mathrm{H}_{2}$ distribution $\left(\mu_{\text {Left }}<\mu_{\text {Right }}\right)$.

\section{Additional computational results}
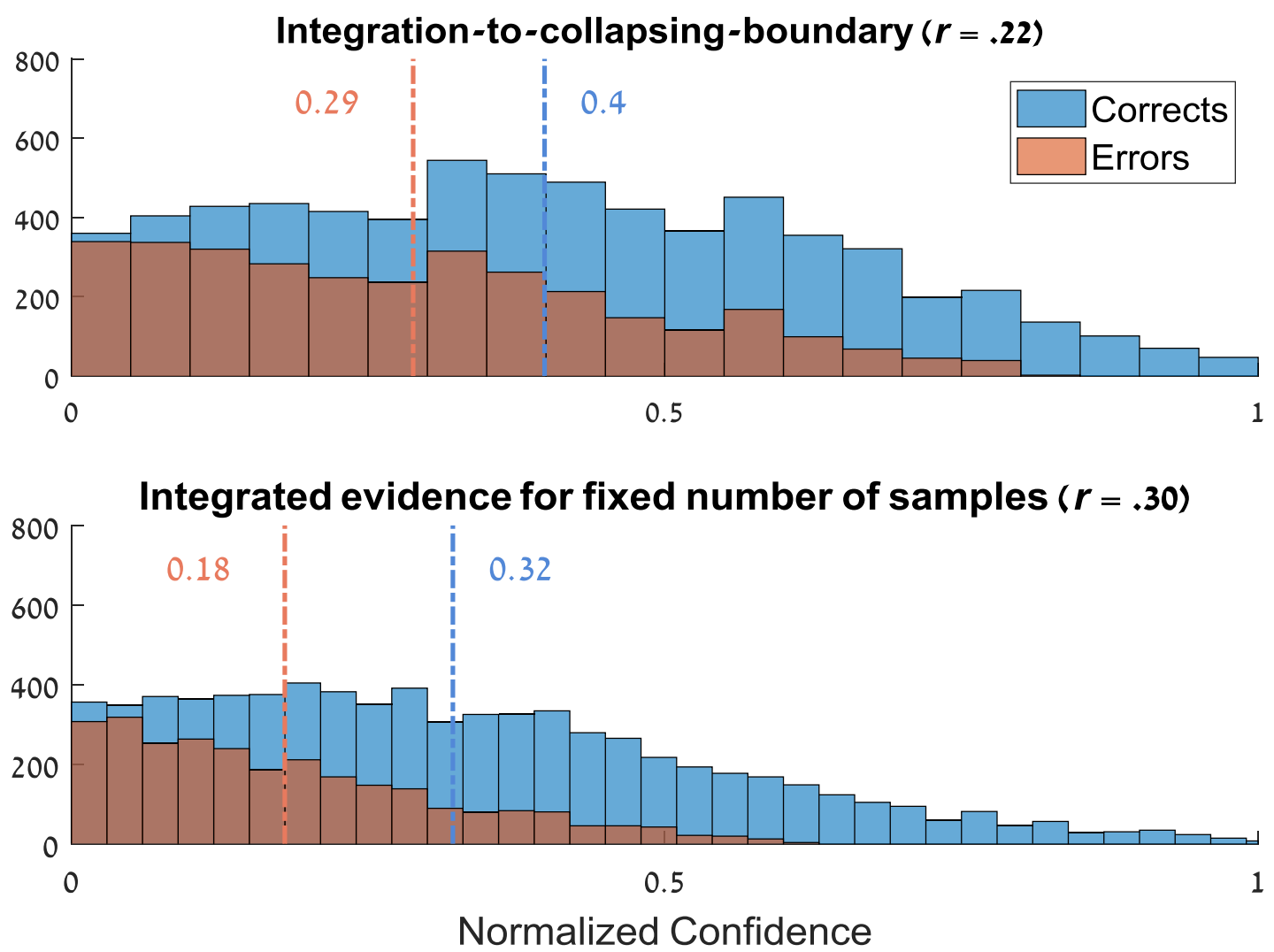
Fig. S2. Confidence distributions for free response and interrogation protocols. As in Fig 3, but here the fixed boundary is replaced with a collapsing one (Hawkins et al., 2015). The figure shows the distribution of confidence levels for correct (blue) and incorrect (red) responses.

\section{Computational methods}

\section{Model-selection (choice)}

For each participant in session 1 we fitted 3 models to their decisions (conditional on actual evidence samples and on RT):

i) Diffusion with fixed boundary (the evidence difference was integrated subject to external noise) to two fixed boundaries.

ii) Diffusion with collapsing boundary (we used a 3 parameter Weibull parametrization of the collapsing boundary (Hawkins et al., 2015):

$$
u(t)=a-\left[1-\exp \left(-\left(\frac{t}{\lambda}\right)^{k}\right)\right] \cdot\left(a-a^{\prime}\right)
$$

iii) Vickers (1970) model. Here if the difference is favors the left stream it is accumulated in a leftaccumulator, and if it favors the right stream it is accumulated in a separate right-accumulator. RT is determined by the race of the two accumulators.

All the models included additional external noise (Normally distributed). Models ii) and iii) allow accounting for positive confidence-resolution in session 1 (free response). For session 2 (interrogation) we assumed that decisions were based on signal-detection theory, which is applied to the total-evidence (criterion 0). The model classification strongly favors model ii (Diffusion with collapsing boundary) for the free response session. Only 1 subject was classified as better supported by the Vickers model (see Table 3 in Supplement).

\section{Model-selection (confidence)}

We used several models to predict confidence using a regression analysis. The models were consisted of different combinations of a few predictors (See Tables 4 and 5 in Suppl for model comparison):

1. Accumulated evidence $=\sum_{i=1}^{n} x_{i}-y_{i}$

Where for each sample $i, x=$ the sequence selected by the participant; $y=$ the unselected sequence.

Leaky accumulated evidence $=\sum_{i=1}^{n}\left(x_{i}-y_{i}\right) \lambda^{\mathrm{n}-\mathrm{i}}$

2. RT (number of samples in each trial) 
3. Last item evidence $=x_{n}-y_{n}$

4. Last item evidence- $1=x_{n-1}-y_{n-1}$

5. Stop boundary point. Each participant's data was fitted to a collapsing boundary diffusion model. This predictor is correlated with RT and indicates the point on the boundary in which the participant took the decision in every trial.

6. Split evidence $=b_{1} x-b_{2} y$

We used BIC measure fits for model classification.

\section{Additional data in Experiment 2}

\section{i) RT for correct and incorrect trials}

To understand sources of confidence resolution in session 1 we tested if correct trials were faster than incorrect trials; we created two RT histograms (for correct and incorrect trials) for each participant and averaged quantiles 10, 30, 50, 70 and 90 across participants. A t-test showed that the median RT was significantly faster for correct trials compared to incorrect trials $(t(34)=4.3 p<0.001)$.

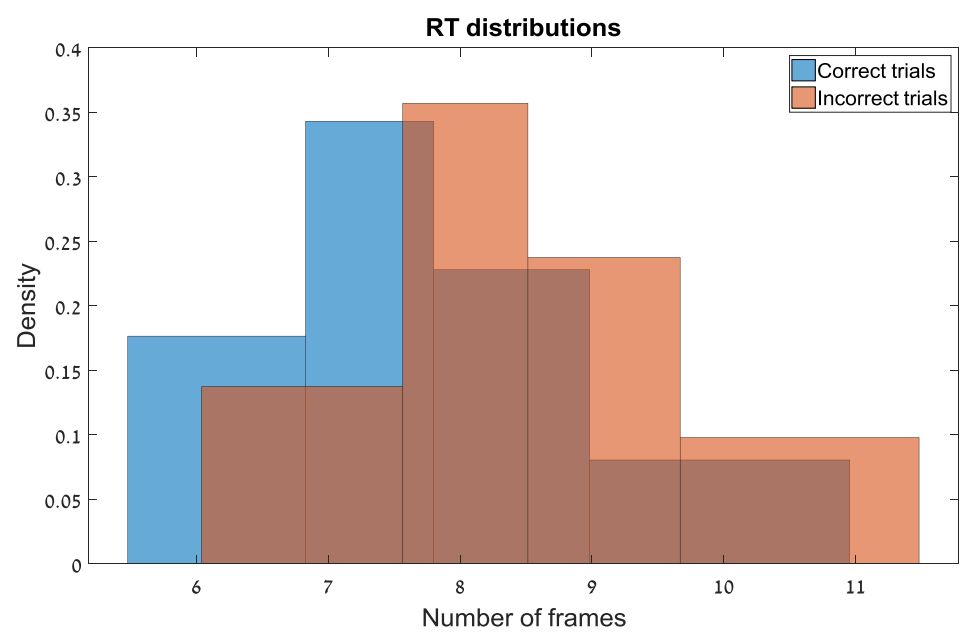

Fig S3. RT distributions (number of frames) for correct and incorrect responses in the free-response session. The faster $R T$ for correct choices explains how a strategy that uses RT to inform confidence can generate some degree of confidence resolution.

ii) Multiple regression of confidence from $R T$ and accumulated evidence in Exp-2. 


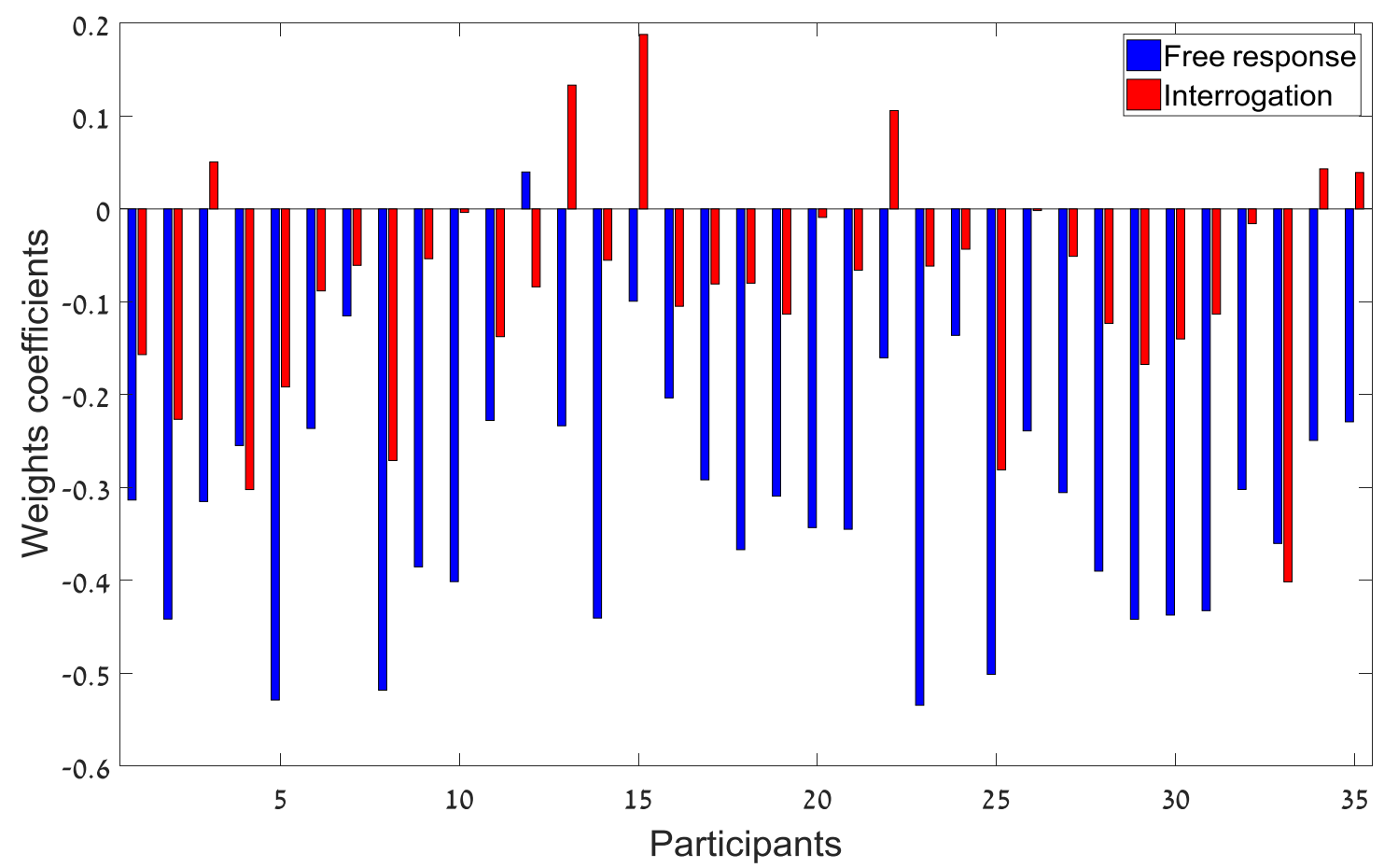

Fig S4. RT coefficients as a confidence predictor in a multiple regression that includes accumulated evidence as a second predictor, for each participant in the free-response and the interrogation session in Experiment 2. Group coefficients is -.31 (SD=.13) for the free response, and $-0.08(S D=.12)$ for the interrogation task.

iii) Reversed correlation for selected/unselected input with choice in Exp-2, interrogation session.

To examine if participants rely on an implicit boundary in their choices in the interrogation session (Kiani et al., 2008), we carried out a reverse correlation of the presented evidence based on choices made in all trials (see Kiani et al., for methological details). While implicit boundary models predict primacy (early evidence has more impact on the decision, as late evidence may arrive after boundary is reached), the signal detection model based on integrated evidence (or leaky integrated evidence) predicts either uniform temporal weights or recency. As shown in Fig S4, the data shows no primacy and possibly a small recency. 

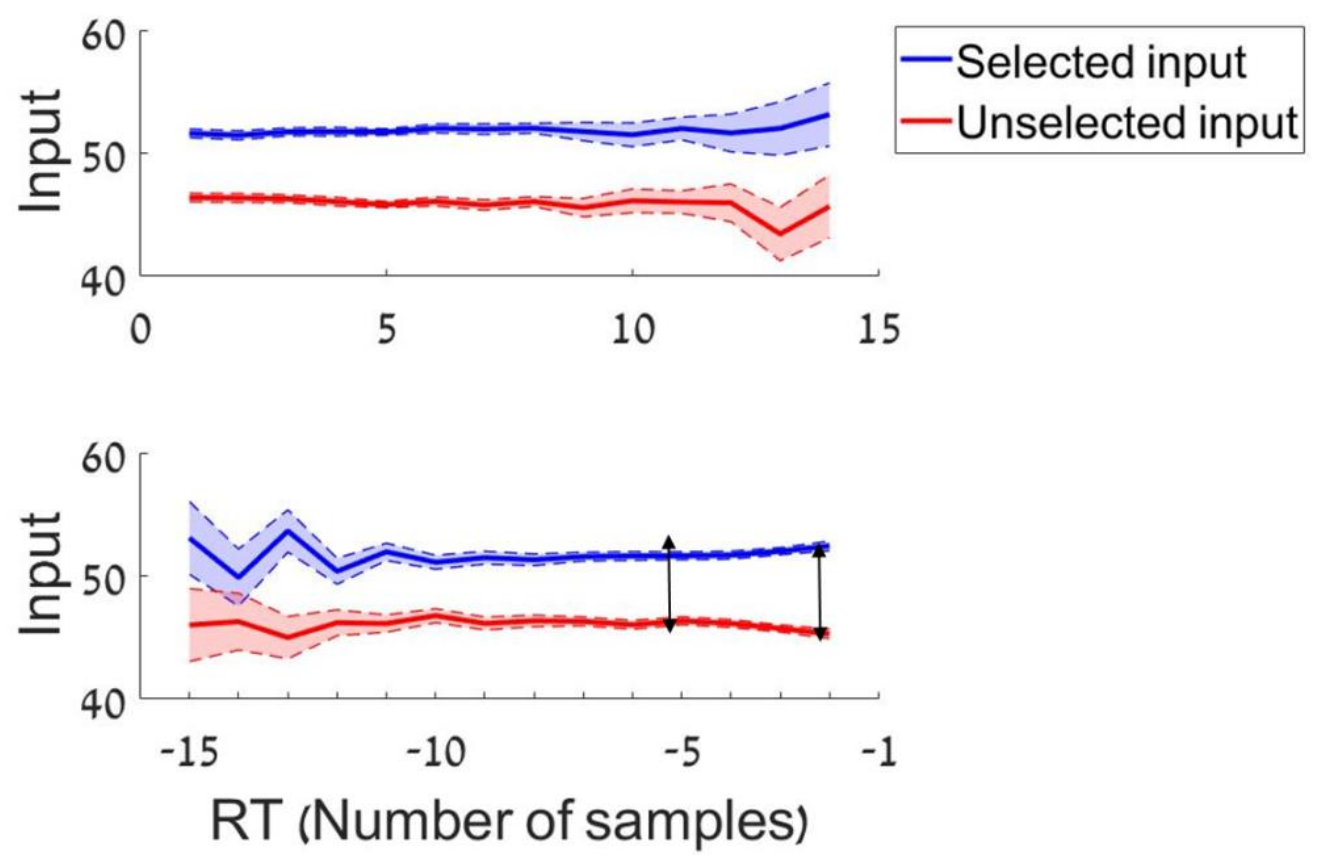

Fig S5. Reverse correlation of choices based on the two evidence streams (red-selected and blueunselected) in Experiment 2, session-2. The lines correspond to the average of the two streams across all trials and the shade corresponds to confidence intervals. Top panel: the evidence streams are time locked to the start of the trial. Bottom panel, the evidence streams are time locked to the response. The data indicates a small recency (the two black arrows have the same height).

\section{Choice models comparison:}

Table-3: Free-response task

\begin{tabular}{|c|c|c|c|}
\hline Subject & Diffusion model Fixed & Diffusion model Collapsed & Accumulator model \\
\hline 1 & 1108 & $\mathbf{8 0 0}$ & 1022 \\
\hline 2 & 947 & $\mathbf{7 8 4}$ & 893 \\
\hline 3 & 1073 & $\mathbf{8 3 1}$ & 1074 \\
\hline 4 & 1041 & $\mathbf{8 3 5}$ & 1012 \\
\hline 5 & 908 & $\mathbf{7 8 3}$ & 849 \\
\hline 6 & 1281 & $\mathbf{1 0 5 7}$ & 1318 \\
\hline 7 & 958 & $\mathbf{9 0 7}$ & 1044 \\
\hline
\end{tabular}




\begin{tabular}{|c|c|c|c|}
\hline 8 & 1063 & $\mathbf{8 1 9}$ & 1010 \\
\hline 9 & 1042 & $\mathbf{8 6 3}$ & 1041 \\
\hline 10 & 1053 & $\mathbf{7 7 3}$ & 869 \\
\hline 11 & 981 & $\mathbf{8 4 8}$ & 977 \\
\hline 12 & 920 & $\mathbf{8 0 8}$ & 946 \\
\hline 13 & 1028 & $\mathbf{8 1 3}$ & 1037 \\
\hline 14 & 981 & $\mathbf{7 6 2}$ & 931 \\
\hline 15 & 1044 & $\mathbf{8 8 7}$ & 1088 \\
\hline 16 & 943 & $\mathbf{7 9 9}$ & 931 \\
\hline 17 & 981 & $\mathbf{8 7 7}$ & 995 \\
\hline 18 & 778 & 699 & $\mathbf{6 7 5}$ \\
\hline 19 & 1006 & $\mathbf{7 4 2}$ & 956 \\
\hline 20 & 933 & $\mathbf{7 5 7}$ & 909 \\
\hline 21 & 998 & $\mathbf{7 3 0}$ & 841 \\
\hline 22 & 1091 & $\mathbf{8 6 1}$ & 1104 \\
\hline 23 & 1006 & $\mathbf{8 1 8}$ & 929 \\
\hline 24 & 1091 & $\mathbf{7 5 7}$ & 977 \\
\hline 25 & 983 & $\mathbf{8 6 5}$ & 906 \\
\hline 26 & 976 & $\mathbf{8 2 2}$ & 1065 \\
\hline 27 & 973 & $\mathbf{7 8 9}$ & 956 \\
\hline 28 & 947 & $\mathbf{7 3 1}$ & 845 \\
\hline 29 & 872 & $\mathbf{6 8 0}$ & 817 \\
\hline 30 & 1020 & $\mathbf{7 4 1}$ & 912 \\
\hline 31 & 858 & $\mathbf{7 0 2}$ & 856 \\
\hline 32 & 1015 & $\mathbf{6 0 1}$ & 1023 \\
\hline 33 & 953 & $\mathbf{8 5 3}$ & 979 \\
\hline 34 & 1047 & $\mathbf{7 8 7}$ & 1007 \\
\hline 35 & 1094 & $\mathbf{8 3 8}$ & 1067 \\
\hline Average & $\mathbf{1 0 0 0}$ & $\mathbf{8 0 1}$ & $\mathbf{9 6 7}$ \\
\hline & & & \\
\hline
\end{tabular}

\section{CONFIDENCE}

Free response

Table-4

\begin{tabular}{|l|c|c|c|}
\hline \multicolumn{1}{c}{ Model } & BIC & R & (-2)*LL \\
\hline LastItemEvidence_RestEvidence_RT & 346.50 & 0.57 & 326.88 \\
\hline LastItemEvidence_RT & 352.42 & 0.53 & 337.71 \\
\hline SplitLastItem_RT & 354.97 & 0.53 & 335.35 \\
\hline LastItemEvidence_StoppingPointBoundary & 355.14 & 0.51 & 340.42 \\
\hline AccumulatedEvidence_RT & 355.30 & 0.51 & 340.59 \\
\hline LastItemEvidence_StoppingPointBoundary_RT & 355.70 & 0.53 & 336.08 \\
\hline LeakyIntegratedEvidence & 356.86 & 0.51 & 342.00 \\
\hline
\end{tabular}




\begin{tabular}{|l|c|c|c|}
\hline SplitLastItem_StoppingPoint & 357.64 & 0.52 & 338.02 \\
\hline LastItemEvidence_RestEvidence_RT & 360.60 & 0.48 & 345.89 \\
\hline LastItemEvidence_LastItemEvidence-1_Rest & 361.79 & 0.50 & 342.17 \\
\hline AccumulatedEvidence & 368.16 & 0.41 & 358.35 \\
\hline SelectedSamples_UnselectedSamples & 374.37 & 0.50 & 340.04 \\
\hline
\end{tabular}

\section{Interrogation}

Table-5

\begin{tabular}{|l|c|c|c|}
\hline \multicolumn{1}{|c}{ Model } & BIC & R & (-2)*LL \\
\hline AccumulatedEvidence & 357.65 & 0.48 & 347.84 \\
\hline LeakyIntegratedEvidence & 358.3 & 0.50 & 343.59 \\
\hline LastItemEvidence_RestEvidence & 359.32 & 0.49 & 344.6 \\
\hline AccumulatedEvidence_RT & 359.86 & 0.49 & 345.14 \\
\hline LastItemEvidence_RestEvidence_RT & 361.84 & 0.50 & 342.23 \\
\hline LastItemEvidence_LastItemEvidence-1_RestEvidence & 362.42 & 0.50 & 342.81 \\
\hline LastItemEvidence_LastItemEvidence-1_RestEvidence_RT & 361.13 & 0.50 & 340.61 \\
\hline LastItemEvidence_RT & 389.41 & 0.25 & 374.70 \\
\hline SelectedSamples_UnselectedSamples & 392.82 & 0.37 & 358.50 \\
\hline SplitLastItem_RT & 393.08 & 0.25 & 373.47 \\
\hline
\end{tabular}

In the free response session, we see that the models that best account for confidence include RT, and the Last-item evidence (in some of the trials the latter involves post-decision integration). Both RT and post-decision integration can indicate correct choices and can mediate the smaller confidence resolution in the free response session. We also examined if there is an asymmetry in the weight given to chosen vs. unchosen evidence in confidence (Zylberberg, Barttfeld \& Sigman, 2012). While the weight appeared higher for the chosen evidence this was not significant and it did not win in terms of BIC. In the interrogation session, the accumulated evidence appears to be the best predictor for confidence. Leaky-integration comes second (in terms of BIC, but higher in Log-Likelihood).

\section{Wagering predictions}

We consider a wagering version of our task. In this wagering version, the participants would view samples from the same distributions as in our experiments [see Fig. 2; normal distributions: Left $N\left(52,10^{2}\right)$ and Right $\left.\sim N\left(46,10^{2}\right)\right]$ and choice accuracy is potentially rewarded in the following way: 1 point for each correct response, -3 points for each incorrect response. The subjects, however, have the 
possibility to wager on their choice based on their internal decision confidence. If they feel confident they wager (which means that their potential reward will count and accumulate towards the total reward), while if they are not confident they do not wager (which means that no reward will be accumulated on that trial). ${ }^{5}$ Since this is based on a binary level of confidence, we transformed modelconfidence to two levels based on a median split (across trials). Thus, in each trial a subject could accumulate the following number of points: i) +1 , in case of a correct choice and confidence was higher than median confidence, ii) -3 in case of an incorrect choice and confidence which was higher than median confidence, iii) 0 , in case confidence was lower than the median confidence. The total wagering score is the total number of accumulated points over all trials.

We compared three choice and confidence mechanisms on their wagering performance. The mechanisms we considered are: i) integration to a fixed boundary (for choice), followed by 1 postdecision sample (for confidence), ii) integration to a collapsing boundary (for choice and for confidence), iii) integration over a fixed number of samples (using SDT for both choice and confidence; see Fig S1). The boundaries (in the first two models, and the number of samples in the third model, were selected so as to obtain an equal average number of samples (RT), across models (this was selected to be 7, which is in the range of experimental data).

The algorithm for choice in each strategy was simulated by sampling pairs of values from the two distributions and accumulating the differences of each pair $\left(\sum_{i=1}^{N} L i-R i\right)$. Trials were terminated when the accumulated evidence reached a predefined decision boundary (integration to fixed lcollapsing boundaries) or after a fixed number of samples (fixed timer strategy). In order to equalize the mean number of samples for each strategy ( 7 samples), the fixed boundary was set to 67 and the collapsing boundary was defined by the Weibull function:

$$
u(t)=a-\left[1-\exp \left(-\left(\frac{t}{\lambda}\right)^{k}\right)\right] \cdot\left(a-a^{\prime}\right)
$$

where the intercept (a) was set to 205 , the asymptote ( $\left.a^{\prime}\right)$ was set to 20 and the scale parameter $(\lambda)$ was set to 6.8 .

The confidence mechanism was simulated exactly the same as described above in the predictions of confidence resolution section (extra sample for integration to fix boundary and total evidence for collapsing boundary and random timer strategies). As shown in Fig. S6, a collapsing boundary mecha-

\footnotetext{
${ }^{5}$ Since the rewards are asymmetric with losses higher than gains, it is not worth to wager on trials on which one is at chance of being correct.
} 
nism (dark blue) which gives a compromise between the fixed boundary and fixed number of samples mechanisms (light blue and red, respectively), in accuracy (left panel) and in confidence resolution (middle panel), results here in the highest wagering score (right panel). This is because wagering combines accuracy and confidence resolution.

\section{Fixed boundaries $\quad$ Collapsing boundaries} Fixed number of samples
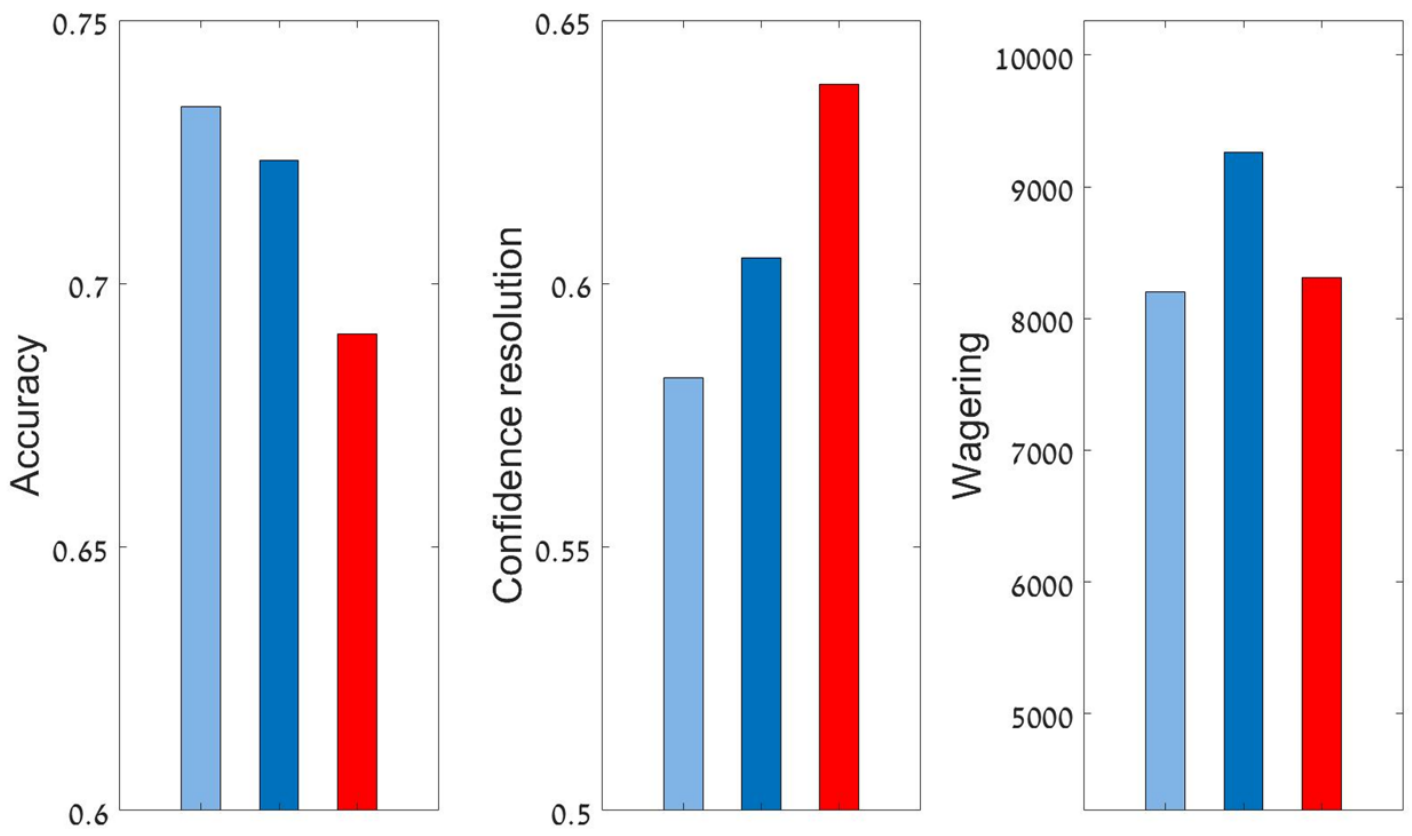

Fig. S6. Simulation for the three strategies: i) Integration to fixed boundaries ii) Integration to collapsing boundaries iii) Fixed timer. Left panel. Accuracy rate for each strategy. Middle panel. Confidence resolution for all strategies measured in type 2 AUROC. Right panel. Wagering score for each strategy which takes into consideration both accuracy and confidence resolution.

\section{Control experiment}

The aim of this study was to rule out a possible confound of a practice effect in metacognition performance, which could give an alternative explanation for the performance improvement in experiment 3 .

\section{Methods}

Participants. 35 undergraduates from Tel-Aviv University (21 females; age: $M=22$, range 21-26 years) participated in the experiment. The participants received course credit in exchange for taking part in the experiment. The experiment was approved by the ethics committee at TAU. 
Procedure and design. The task was similar to experiment 3 with one exception: I) The two sessions were a self-terminated task (Free response). The number of trials for each session was 80 (same as in experiment 3). In this case, if the metacognition improvement was due to a practice effect, we would expect an improved metacognition performance in the second session, in this experiment as well. See Results in the main text (Discussion section). 